\title{
Ensemble Sensitivity Analysis of Tropical Cyclone Intensification Rate during the Development Stage
}

\author{
CHIH-Chi Hu AND CHUN-ChIEH Wu \\ Department of Atmospheric Sciences, National Taiwan University, Taipei, Taiwan
}

(Manuscript received 19 July 2019, in final form 29 July 2020)

\begin{abstract}
Ensemble sensitivity analysis based on convective-permitting ensemble simulations is used to understand the processes associated with tropical cyclone (TC) intensification under idealized conditions. Partial correlations between different variables and the future TC intensification rate, with the effect of intensity removed, are used to identify the sensitive factors. It is found that the equivalent potential temperature $\left(\theta_{e}\right)$ in the region from the radius of maximum wind (RMW) to 3 times the RMW below $2 \mathrm{~km}$ (hereafter, the sensitive region) has the largest correlation (over 0.7) with 2.5-h intensity change. It is found that higher $\theta_{e}$ in the sensitive region is associated with not only a stronger updraft but also an inward shift of vertical motion in the mid- to upper eyewall. This suggests that higher $\theta_{e}$ just outside the RMW is favorable to TC intensification not only because of the larger amount of the heating, but also due to the heating location that is closer to the center. Trajectory analysis shows that the parcels in the sensitive region are mainly from the boundary layer inflow and the midlevel inflow. It is found that when the outer rainband is active, the midlevel inflow becomes stronger and is able to bring more low $-\theta_{e}$ air into the boundary layer, and the $\theta_{e}$ radially inward to the rainband decreases. Verification experiments justify that higher $\theta_{e}$ around the RMW to 3 times the RMW is favorable to TC intensification, while higher $\theta_{e}$ away from 5 times the RMW is shown to be unfavorable for TC intensification.
\end{abstract}

KEYWORDS: Tropical cyclones; Ensembles; Model output statistics; Numerical analysis/modeling

\section{Introduction}

The accuracy in tropical cyclone (TC) intensity forecasting is still behind that of track forecasting (e.g., DeMaria et al. 2014; Yamaguchi et al. 2017). Recent studies have indicated that the initial intensity error and the specification of inner-core moisture are the two major sources of TC intensity forecast errors (Emanuel and Zhang 2016, 2017).

Many studies utilizing the statistics from the ensemble Kalman filter (EnKF) have also shown that the subsequent TC intensity is sensitive to the TC structures that are strongly dependent on initial intensity (e.g., Torn and Cook 2013; Brown and Hakim 2015; Emanuel and Zhang 2017; Liu et al. 2018; Minamide and Zhang 2018; Nystrom et al. 2018). These results highlight the importance of accurately constraining the initial $\mathrm{TC}$ intensity in order to reliably predict future TC intensity.

To understand the dynamics of TC intensification, it is beneficial to also identify factors to which the TC intensification rate is sensitive. Emanuel and Zhang (2017) showed that the ensemble with initial intensity error but the same innercore moisture has a very similar intensification rate at least during the first 12-24 h (their Fig. 6a). However, the ensemble with only initial inner-core moisture error yet with the same initial intensity shows a large spread of intensification rate in the first $12 \mathrm{~h}$. In addition, it is shown in their Fig. 10 that the intensity error due to the initial inner-core moisture error grows very fast and becomes saturated quickly. This suggests that the moisture can play an important role in the transient TC intensification rate, which is especially important, for example, when the TC is close to landfall (Kimball 2006).

Corresponding author: Chun-Chieh Wu, cwu@as.ntu.edu.tw
The importance of inner-core moisture has also been demonstrated in many studies using different methods. Minamide and Zhang (2018) assimilated all-sky infrared satellite radiances from Himawari-8 to study the rapid intensification (RI) of Super Typhoon Soudelor (2015) and demonstrated that the updated axisymmetric component of water-related variables alone can help accurately capture the early-stage intensification rate (their Fig. 10), leading to an improvement of the RI onset time as well. Some studies have utilized the adjoint model to demonstrate similar sensitivity in the subsequent TC intensity. These studies have shown the subsequent TC intensity is much more sensitive to the mass field (moisture and temperature) than to the wind field (e.g., Ito et al. 2011; Doyle et al. 2012). Note that since there is no inherent initial uncertainty in TC intensity by using the adjoint model, the subsequent perturbation TC intensity is equivalent to the perturbation TC intensification rate.

Although many studies have agreed on the importance of moisture on the TC intensification rate, the location of this sensitivity has seldom been examined. The inner-core moisture is known to be favorable for TC intensification, as the latent heat release from the moisture is used as the major source of energy in TC (e.g., Emanuel 1991). However, studies have shown that the moisture in the outer region is, on the contrary, unfavorable for transient TC intensification (e.g., Kimball 2006; Ito et al. 2011; Ying and Zhang 2012).

Therefore, the purpose of this study is to systematically identify the sensitivity of TC intensification rate to various variables, specifically focusing on the moisture related variables and their spatial pattern. Since many of the previous studies have examined the sensitivity of TC intensification in real cases, in which the environmental factors are difficult to 
(a)

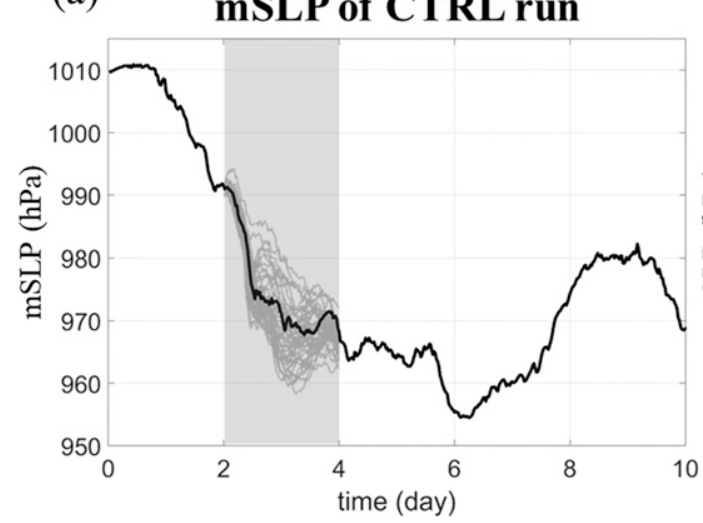

(b) mSLP of ensemble members

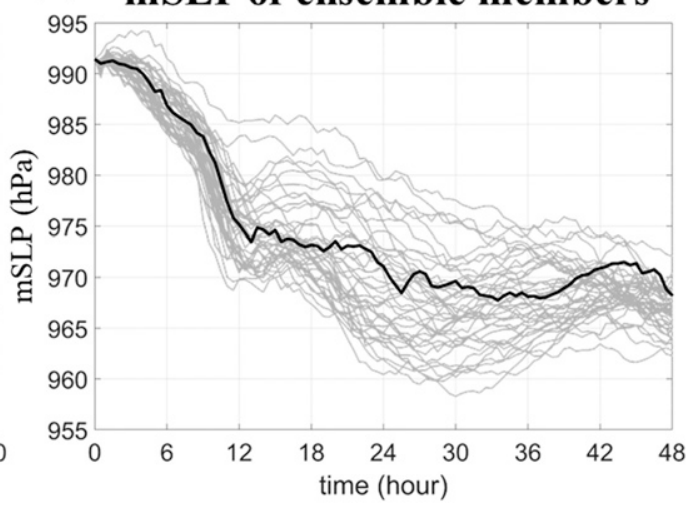

FIG. 1. The time series of the minimum sea level pressure. (a) The 10-days integration for CTRL run (black line) and 2-days integration for ensemble members (gray line). (b) The enlarged period of the shaded region in (a).

control, we are motivated to identify the sensitive factors under idealized conditions. An idealized framework using an axisymmetric model has already been shown to be useful in understanding the sensitivity to TC intensity (e.g., Ito et al. 2011; Alland et al. 2017). Considering the importance of convection in TC intensification, a full-physics and convection-permitting $3 \mathrm{D}$ model is used to examine the sensitivity of TC intensification rates.

The remainder of this paper is organized as follows: Section 2 describes the model settings and the method for the sensitivity estimation. Section 3 analyzes partial correlation results and identifies the variable to which the TC intensification rate is most sensitive. Section 4 discusses the response of a TC to the sensitive variable and the factors leading to variations of the sensitive variable. Section 5 discusses the result of a set of verification experiments. Section 6 concludes the findings of this study.

\section{Models and methods}

\section{a. Model configurations}

The Weather Research and Forecasting (WRF) Model (version 3.6.1) is used to simulate TC-like vortex evolution in an idealized environment. The horizontal resolution is $2.5 \mathrm{~km}$ $(500 \times 500$ grid points $),{ }^{1}$ and 40 vertical eta levels are used. The doubly periodic boundary condition is used. The initial vortex structure is based on Rotunno and Emanuel (1987), which is an axisymmetric vortex with the radius of maximum wind (RMW) set as $50 \mathrm{~km}$, and the maximum wind set as $15 \mathrm{~m} \mathrm{~s}^{-1}$. The sea surface temperature is fixed at $300 \mathrm{~K}$ uniformly across the area

\footnotetext{
${ }^{1}$ Considering $1250 \mathrm{~km} \times 1250 \mathrm{~km}(500 \times 500$ grid points $)$ may be small for simulating a TC, we also conduct an experiment with larger domain size of $2500 \mathrm{~km} \times 2500 \mathrm{~km}(1000 \times 1000$ grid points $)$. The general vortex structure remains similar using a larger domain as compared to the smaller domain, which can be attributed to the small size of initial vortex. To save computational resources, $500 \times$ 500 grid points is used for the following experiments.
}

during all integration time. The Jordan sounding (Jordan 1958) is used as the initial atmospheric temperature and humidity profile. The simulation is on an $f$ plane $\left(f=5.0 \times 10^{-5} \mathrm{~s}^{-1}\right)$ without terrain. The Goddard GCE scheme (Tao et al. 1989) is used for microphysics parameterization, the Yonsei University (YSU) planetary boundary layer scheme (Hong et al. 2006) is used for boundary layer parameterization, the Rapid Radiative Transfer Model (RRTM) scheme (Mlawer et al. 1997) is used for longwave radiation, and the Dudhia scheme (Dudhia 1989) is used for shortwave radiation. Due to the high horizontal resolution, the cumulus parameterization scheme is omitted here.

\section{b. Generating ensembles}

A control run (CTRL) using the settings described in section $2 \mathrm{a}$ is first integrated for 10 days, and the minimum sea level pressure (mSLP) for the CTRL run is shown in Fig. 1a. To examine the sensitivity of intensification rate, perturbations are added to the onset time of a significant intensification period, which is the day- 2 output of the CTRL run.

Unlike a real-case study, in which we can obtain reasonable perturbation patterns informed by available observational data and ensemble data assimilation techniques (e.g., Poterjoy and Zhang 2011), an idealized simulation does not provide the background error covariance of TC structure through the same way. Therefore, we propose a new way to create perturbations in this study. For simplicity, we only consider wavenumber-0 perturbations, which means the perturbations are ringlike. This is reasonable since the environmental conditions in our scenario are isotropic relative to the TC center. Furthermore, Minamide and Zhang (2018) have shown the importance of wavenumber 0 moisture structure in TC intensity forecasting. Following Emanuel and Zhang (2017), which indicated the important role of moisture distribution in TC intensification rate, we use water vapor $\left(q_{v}\right)$ as the only perturbed variable.

Previous studies using $q_{v}$ as a perturbed variable have utilized the white noise patterns with the magnitude selected from a uniform distribution (e.g., Van Sang et al. 2008; Tao and Zhang 2014). In this study, we create perturbation with spatial 
(a)
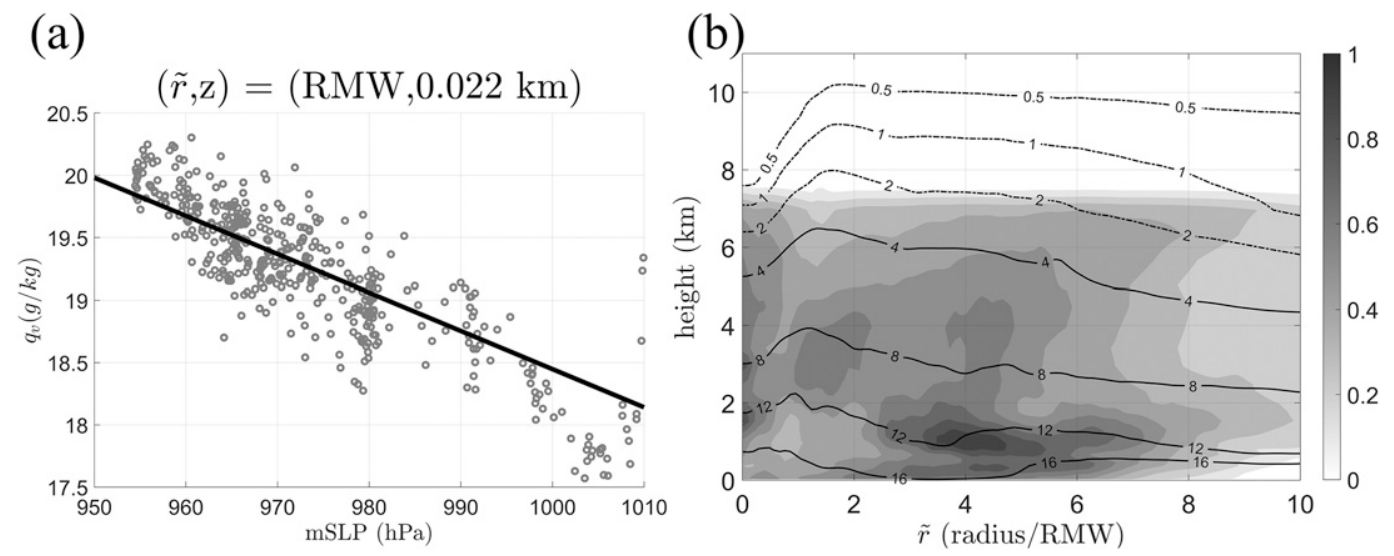

FIG. 2. (a) The demonstration of perturbation generation. The simple linear regression is at $\tilde{r}=\mathrm{RMW}$ and $z=$ $0.023 \mathrm{~km}$, the $x$ axis is the minimum sea level pressure and the $y$ axis is the water vapor mixing ratio $\left(\mathrm{g} \mathrm{kg}^{-1}\right)$. The circles are the output from the CTRL run, and the line is the regression line. The $q_{v}$ perturbation at $(\tilde{r}, z)=(\mathrm{RMW}$, $0.023 \mathrm{~km}$ ) is defined as the deviation of the dot from the line. (b) The standard deviation (shaded, $\mathrm{g} \mathrm{kg}^{-1}$ ) of the water vapor perturbations within the ensemble members and the water vapor mixing ratio (contoured, $\mathrm{g} \mathrm{kg}^{-1}$ ) of the CTRL run. The $x$ axis is the radius normalized by RMW and the $y$ axis is the height.

patterns controlled to some extent by the underlying dynamics. We use all the output times ${ }^{2}$ in the CTRL run as the samples, and set up a linear regression model between the $q_{v}$ distribution and TC intensity (in terms of the mSLP) at each radius and height. The residual for each sample is taken as a $q_{v}$ perturbation $\left(q_{v}^{\prime}\right)$. Since the TC structure changes significantly from a nascent stage to a mature stage (the RMW contracts as the TC intensifies), we normalize the azimuthally averaged water vapor $q_{v}(r, z, t)$ for all the samples by their $\mathrm{RMW}^{3} q_{v}(\tilde{r}, z, t)(\tilde{r}$ is the radius normalized by the RMW, $r=\tilde{r} \times \mathrm{RMW})$ when building the regression model. The regression model at each $\tilde{r}$ and height $z$ can be written as

$$
q_{v}(\tilde{r}, z, t)=\alpha(\tilde{r}, z)+\beta(\tilde{r}, z) \operatorname{mSLP}(t)+q_{v}^{\prime}(\tilde{r}, z, t),
$$

where $\alpha$ and $\beta$ are the intercept and the slope of the regression line, respectively. Figure 2a shows one of the regression models at the RMW and an altitude of $0.023 \mathrm{~km}$. Each dot in Fig. $2 \mathrm{a}$ corresponds to an output time in the CTRL run. Since we have 480 output times from the CTRL run, $480 q_{v}^{\prime}$ patterns are created by the regression model.

Examination of all the $q_{v}^{\prime}$ patterns show that there are coherent structures representing TC structural changes (figures not shown). Since the coherent structures do not change significantly within a few hours, if the $q_{v}^{\prime}$ pattern are chosen with small temporal separation, the diversity of the initial conditions within the ensemble can be small. Therefore, $40 q_{v}^{\prime}$ patterns are randomly selected as the initial ensemble perturbations, but with enough temporal separation between each ensemble member to ensure diversity.

\footnotetext{
${ }^{2} \mathrm{We}$ obtain an output every $0.5 \mathrm{~h}$, and the integration time for CTRL run is 10 days, so the sample size in the regression model is 480 .

${ }^{3}$ The RMW is defined as the average RMW below $0.5 \mathrm{~km}$.
}

The standard deviation of the 40 selected perturbations (Fig. 2b) are less than $10 \%$ of the mean value. These $40 q_{v}^{\prime}$ patterns are added to the $48 \mathrm{~h}$ output from CTRL run, and then integrated for 2 days. The mSLP for the ensemble is shown in Fig. 1b. The standard deviation for mSLP within the ensemble at $t=24 \mathrm{~h}$ is $5.18 \mathrm{hPa}$, which is comparable to the current $24 \mathrm{~h}$ TC intensity forecast error. ${ }^{4}$ Given that only small $q_{v}$ perturbation is responsible for a reasonable intensity spread, this result highlights the important role of water vapor distribution in TC intensity especially during the development stage.

\section{c. Partial correlation analysis}

To understand how the differences in current TC structure affect the future TC intensification rate, we remove the effect of current TC intensity from both variables. One reason is that the TC intensification rate can depend on the current intensity. For example, the Statistical Hurricane Intensity Prediction System (SHIPS) uses the initial maximum wind and the difference between the current intensity and the maximum potential intensity as the predictors of future intensity change (e.g., Table 1 in DeMaria et al. 2005). However, this dependency can be quite complicated if we infer the sensitivity using statistical methods. Specifically, the stronger TCs in the sample will have smaller intensification rate than the weaker TCs on

\footnotetext{
${ }^{4}$ Based on the TC intensity forecast error statistics from the National Hurricane Center (for Atlantic basin, 2015-19, https:// www.nhc.noaa.gov/verification/verify4.shtml), the median for the $24 \mathrm{~h}$ forecast error cumulative distribution is $5 \mathrm{kt}(1 \mathrm{kt} \approx$ $\left.0.51 \mathrm{~m} \mathrm{~s}^{-1}\right)$, which corresponds to $3-6 \mathrm{hPa}$ based on Dvorak intensity chart (e.g., Velden et al. 2006) when the mSLP is around $970 \mathrm{hPa}$. Note that the mean $24 \mathrm{~h}$ forecast error is $7.7 \mathrm{kt}$ (https:// www.nhc.noaa.gov/verification/pdfs/OFCL_5-yr_averages.pdf), which is slightly higher than the median. This may be attributed to some extreme cases with large forecast error.
} 
average. This can lead to a biased relationship if the samples only include TCs with a small range of intensity. Although there are some dynamical reasons supporting the statement that stronger TC have smaller intensification rate (e.g., $\mathrm{Xu}$ and Wang 2015,2018), it can be too complicated to distinguish the dynamical effect and sampling issues in our samples. The other reason is that many TC structures are highly dependent on the current intensity. Principal component analysis within all the outputs in our ensemble shows that over $85 \%$ of the total variance of the azimuthally averaged TC structures ${ }^{5}$ is explained by the current TC intensity (figures not shown). In other words, the current TC intensity is a major confounding variable that we should remove from the TC structure and the TC intensification rate.

One way to compare the TC structures with fixed intensity is to compare the initial conditions for the 40 members and their future (e.g., 0-6 h) intensification rate. However, there are two drawbacks: 1) The sample size may be too small for this problem, which can cause large sampling error. 2) It can be difficult to interpret the effect of initial conditions on the intensification rate after several hours (e.g., after $12 \mathrm{~h}$ ) due to nonlinear effects. To increase the sample size and fully utilize the information within the ensemble members, we collect all the outputs from each ensemble member as the samples. There are 96 output times for each ensemble member (48-h integration and an output for every $0.5 \mathrm{~h}$ ), so the sample size is $96 \times$ $40=3840$.

To compare the samples while controlling for differences in intensity, and to examine how their differences in current TC structures affect the future TC intensification rate, partial correlation analysis is used. Partial correlation measures the linear association between two variables $x$ and $y$, with the effect of a set of controlling variables removed. The first-order partial correlation $\rho_{x y . z}$ between $x$ and $y$ with the controlling variable $z$ can be calculated as follows:

$$
\rho_{x y . z}=\frac{\rho_{x y}-\rho_{x z} \rho_{y z}}{\sqrt{1-\rho_{x z}^{2}} \sqrt{1-\rho_{y z}^{2}}},
$$

where $\rho_{x y}$ is the correlation between two variables $x$ and $y$. See Sippel and Zhang $(2008,2010)$ for more interpretations of the partial correlation. Specifically, we calculate the first-order partial correlation $\rho_{X, d \text { mSLP.mSLP }}$ between azimuthally averaged structure of TC at time $t X(\tilde{r}, z, t)$, and the average TC intensification rate during time $[t, t+\tau](d \operatorname{mSLP}(\tau)=$ $-[\operatorname{mSLP}(t+\tau)-\operatorname{mSLP}(t)])$, with the effect of current TC intensity $\operatorname{mSLP}(t)$ removed.

\section{Partial correlation analysis}

The partial correlations between several important variables and the future TC intensification rates are examined in this section. Note that the intensification rate is defined as the

\footnotetext{
${ }^{5}$ The variables representing the TC structures in the principal component analysis include the azimuthally averaged equivalent potential temperature $\theta_{e}$, tangential wind $v_{t}$, radial wind $v_{r}$, and vertical motion $w$.
}

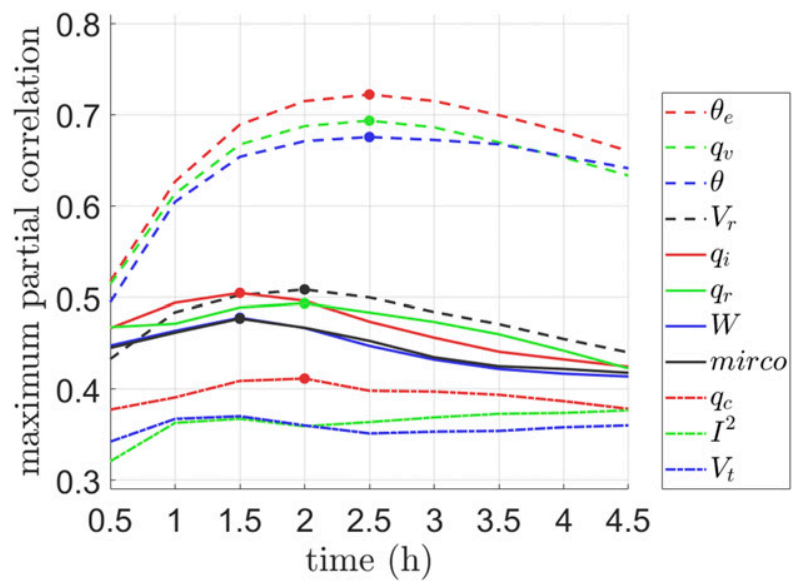

FIG. 3. The spatial maximum partial correlation between several variables and intensification rate averaged between $[t, t+\tau]$. The $x$ axis is the time length $\tau(h)$ for the calculation of average intensification rate, and $y$ axis is the spatial maximum value of the partial correlation. The partial correlation between the intensification rate and the equivalent potential temperature (red dashed line), water vapor mixing ratio (green dashed line), potential temperature (blue dashed line), radial wind (black dashed line), ice mixing ratio (red solid line), rainwater mixing ratio (green solid line), vertical motion (blue solid line), microphysical latent heating (black solid line), cloud water mixing ratio (red dash-dotted line), inertial stability (green dash-dotted lines), and tangential wind (blue dash-dotted line) are shown, and the dot on each line represents the time length $\tau$ when the correlation reaches the maximum.

average intensification rate during $[t, t+\tau]$ (the definition is $d \operatorname{mSLP}(\tau)=-[\operatorname{mSLP}(t+\tau)-\operatorname{mSLP}(t)])$. Different time length $\tau$ for the average intensification rate affects the magnitude of partial correlation, but does not apparently affect the spatial pattern. That is, the spatial maximum (minimum) value also remains similar within a range of $\tau$. We can define the spatial maximum of the magnitude of the partial correlation as

$$
\rho_{X, \max }(\tau)=\max _{\tilde{r}, z}\left|\rho_{X, d \operatorname{mSLP}(\tau) \mathrm{mSLP}}(\tilde{r}, z)\right| .
$$

Figure 3 shows the relation between the maximum value of the correlation $\rho_{X \text {,max }}(\tau)$ with the time length $\tau$ for various variables. Except for the inertial stability and tangential wind, the time $\tau$ for each variable when it reaches the largest magnitude of the correlation can be clearly identified as

$$
\tau_{X, \max }=\underset{\tau}{\operatorname{argmax}}\left[\rho_{X, \max }(\tau)\right]
$$

The $\tau_{X \text {,max }}$ is related to how fast the changes of each variable can affect the TC intensification rate. The fact that the water vapor and equivalent potential temperature result in the largest $\tau_{X \text {,max }}$ implies that they can serve as better precursors for the change of TC intensification rate when the influence of TC intensity is removed. Although we examine the partial correlation between TC intensification rate and each variable independently, variables can be mutually dependent. Their dependencies may be inferred from their $\tau_{X \text {,max }}$. Specifically, variables with larger $\tau_{X \text {,max }}$ may have influence on variables 
(a)

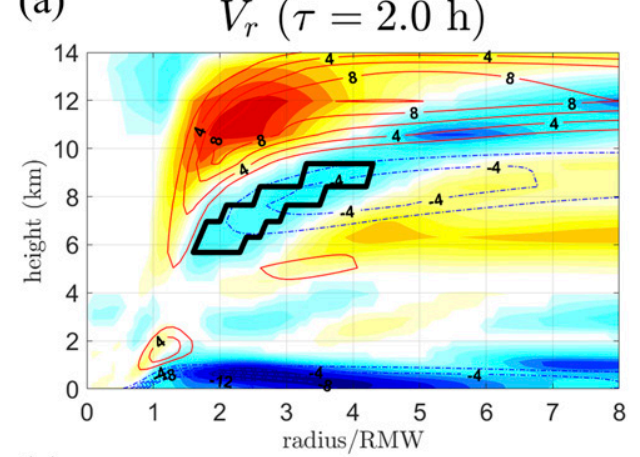

(c)

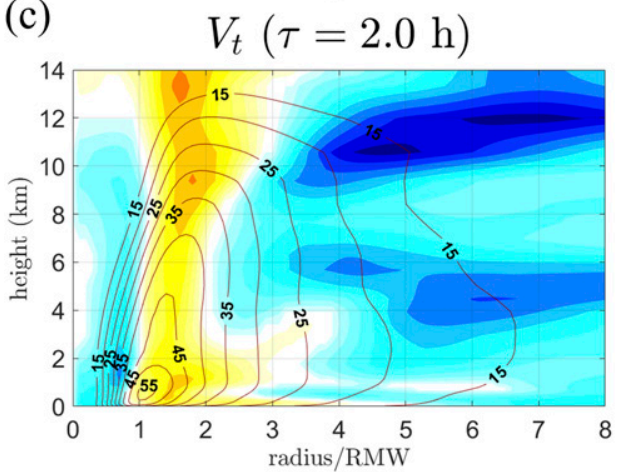

(b)
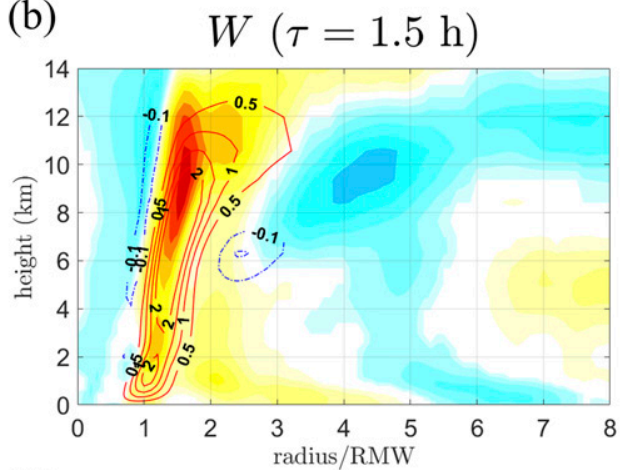

(d)

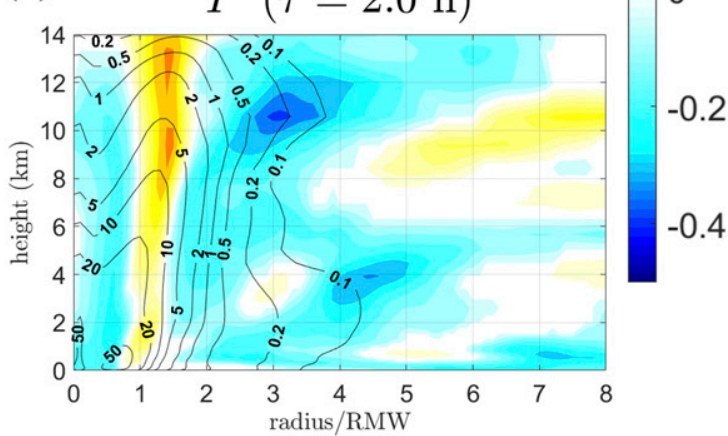

0.4

0.2

0

FIG. 4. The partial correlation (shaded) between intensification rate and different dynamical variables and the sample mean (contoured) for dynamical variables. $\tau$ is the time length for the calculation of average intensification rate, which is based on when the partial correlation reaches the maximum (see Fig. 3). (a) Radial wind ( $\mathrm{m} \mathrm{s}^{-1}$ ). (b) Vertical motion $\left(\mathrm{m} \mathrm{s}^{-1}\right)$. (c) Tangential wind $\left(\mathrm{m} \mathrm{s}^{-1}\right)$. (d) Inertial stability $\left(10^{-6} \mathrm{~s}^{-2}\right)$. The $x$ axis is the radius normalized by RMW and $y$ axis is the height. The black contour in (a) represents the inner edge of the midlevel inflow region.

with smaller $\tau_{X \text {,max }}$. For example, $\tau_{W \text {,max }}$ is smaller than $\tau_{q_{v} \text {,max }}$, suggesting that the larger vertical motion may be a result of larger water vapor. We will focus on the pattern of $\rho_{X, d \mathrm{mSLP}(\tau) \mathrm{mSLP}}$ when it reaches its maximum for each variable in the following discussions. ${ }^{6}$ The following discussions are divided into two parts, focusing on dynamical variables and thermodynamic variables.

\section{a. Dynamical variables}

The sensitivity of TC intensification rate to radial wind $v_{r}$ and vertical motion $w$ (Figs. 4a,b) show that the magnitude of radial inflow below $2 \mathrm{~km}$, radial outflow at 6-14 km, and upward motion from RMW to 2 times the RMW are positively correlated with the intensification rate. This suggests that stronger secondary circulations are associated with greater TC intensification rates (e.g., Rogers et al. 2013). In addition, Fig. 4a shows that there is a region of negative correlation located between midlevel inflow and upper-level outflow. This negative correlation is of interest since the stronger midlevel inflow is expected to bring low equivalent potential temperature $\left(\theta_{e}\right)$ air into the inner core and thereby reducing the intensification rate (Tang and Emanuel 2010, 2012; Didlake and Houze 2013; Molinari et al. 2013), contrary to the result

\footnotetext{
${ }^{6}$ For the inertial stability and tangential wind, $\tau_{X, \max }=2 \mathrm{~h}$ is chosen for the following discussions.
}

obtained from Fig. 4a. We demonstrate here that the negative correlation is actually a result of the spatial correlation with $v_{r}$ in Fig. 5. Examination of the spatial correlations between $v_{r}$ and $w$ (Figs. 5a,b) show that the stronger midlevel inflow (black contour in Fig. 5b) is correlated with many characteristics of a stronger secondary circulation (e.g., stronger boundary layer inflow, stronger midlevel upward motion and stronger upper level outflow). In other words, the midlevel inflow is usually stronger when the secondary circulation is stronger. The role of the midlevel inflow in TC intensification is further discussed in section 4.

Not only the strength, but the location of vertical motion in the eyewall is also important to TC intensification (e.g., Rogers et al. 2013). Figure $4 \mathrm{~b}$ shows that the maximum correlation between $w$ and intensification rate takes place at the upper and inner edge of the maximum mean $w$. This result implies that if the vertical motion is stronger at the inner and upper regions of the eyewall, the TC will have greater intensification rate in the future.

For the correlation with the tangential wind $v_{t}$, Fig. $4 \mathrm{c}$ shows that $v_{t}$ in the mid- to upper eyewall $(7-12 \mathrm{~km})$ has large positive correlation with the intensification rate. The larger $v_{t}$ in mid- to upper eyewall is a result of the stronger secondary circulation and acts to increase the inertial stability in that region (e.g., Chang and $\mathrm{Wu}$ 2017) (Fig. 4d). In addition, there are two regions of negative correlation: one is located between 5 and 8 times the RMW at 3-6 km, and the other between 6 and 8 times the RMW at 9-13 km. This may suggest that TCs with 
(a)

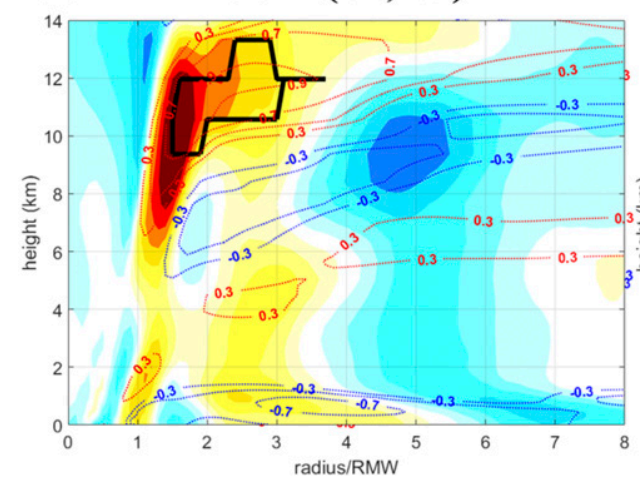

$\operatorname{Corr}(\mathrm{Vr}, \mathrm{W})$

(c)

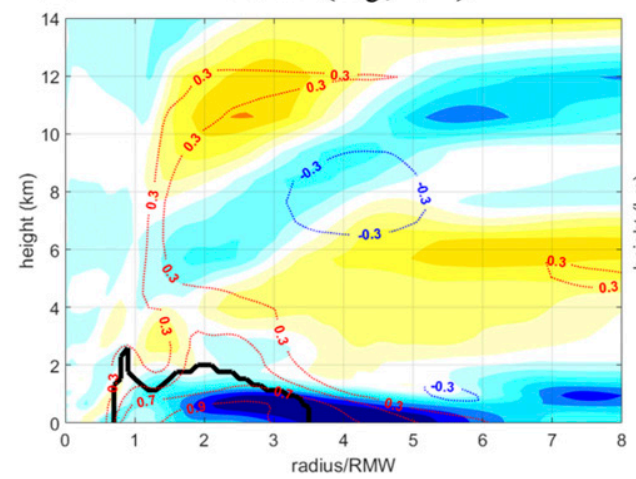

(b) $\quad \operatorname{Corr}(\mathrm{Vr}, \mathrm{W})$

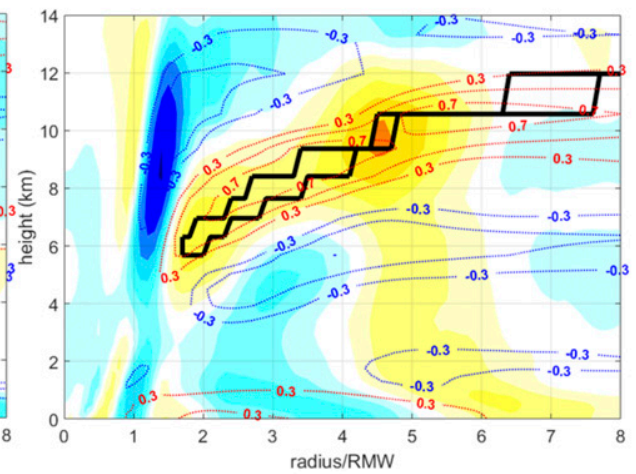

(d) $\quad \operatorname{Corr}\left(\theta_{e}, \mathrm{Vr}\right)$

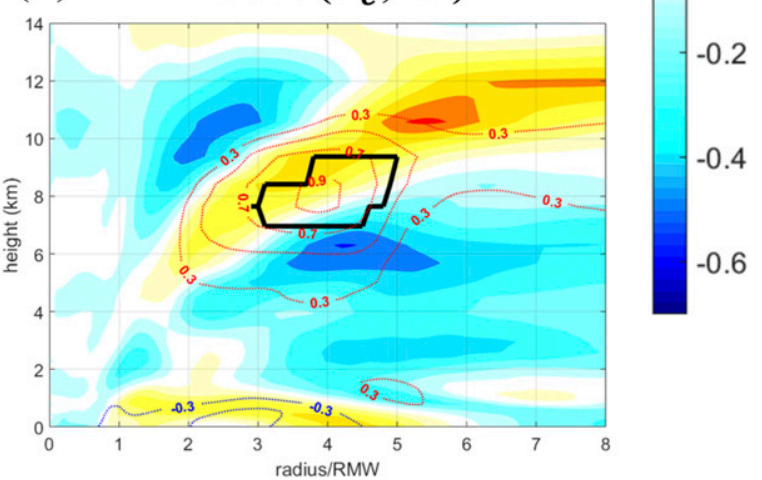

FIG. 5. (a),(b) The spatial correlation between the radial wind averaged in the black contour and the radial wind in space (colored contours) the spatial correlation between the radial wind averaged in the black contour and the vertical motion in space (shading). (c),(d) The spatial correlation between the equivalent potential temperature averaged in the black contour and the equivalent potential temperature in space (colored contours) and the spatial correlation between the equivalent potential temperature averaged in the black contour and the radial wind in space (shading). The $x$ axis is the radius normalized by RMW and the $y$ axis is the height.

smaller size tend to have greater intensification rate (e.g., Carrasco et al. 2014; Tao et al. 2020), while the details merit future investigations.

\section{b. Thermodynamic variables}

The sensitivity of TC intensification rate to $q_{v}$ (Fig. 6a) and $\theta_{e}$ (Fig. 6e) show a similar pattern: ${ }^{7}$ there is a maximum (positive) value from the RMW to 3 times the RMW below $2 \mathrm{~km}$, and a minimum (negative) value located near 3-5 times the RMW at 7-9 km. In addition, there is negative correlations within the exterior of TC (located 5-8 times the RMW) below $4 \mathrm{~km}$ in both Figs. 6a and 6e. This implies that increasing $q_{v}$ (or $\theta_{e}$ ) in the boundary layer is not necessarily favorable for TC intensification, especially in the region outside 5 times the

\footnotetext{
${ }^{7}$ To determine the contribution of variation of $\theta_{e}$, we examine its correlation with $\theta$ and with $q_{v}$ (not shown). We find that the latter is higher, and particularly in the region outside the RMW and below $4 \mathrm{~km}$. The correlation in this region is greater than 0.95 , which means the variation of $\theta_{e}$ in the lower level is almost determined by the variation of $q_{v}$.
}

RMW. The quantitative sensitivity of $q_{v}\left(\right.$ or $\left.\theta_{e}\right)$ in the boundary layer will be further discussed in section 5 . We note that the maximum correlation of $\theta_{e}$ in the region from the RMW to 3 times the RMW below $2 \mathrm{~km}$ (hereafter, the sensitive region) is over 0.7 , which is the largest correlation among all examined variables. Therefore, our result highlights the importance of $\theta_{e}$ (and $q_{v}$ ) just outside the RMW in TC intensification, which supports a number of previous studies (e.g., Miyamoto and Takemi 2013; Wang and Wang 2014; Emanuel and Zhang 2017). We will focus on the effect of $\theta_{e}$ in the sensitive region in the discussion of section 4 .

We also examine the spatial correlation of $\theta_{e}$ and $v_{r}$ (Figs. 5c,d). The $\theta_{e}$ in the sensitive region is negatively correlated with the $\theta_{e}$ in the midlevel (Fig. 5c). In addition, both high $\theta_{e}$ in the sensitive region and low $\theta_{e}$ in the midlevel are associated with a stronger secondary circulation and a stronger midlevel inflow (Figs. 5c,d), i.e., there is an optimal intensifying mode within the ensemble featuring: stronger secondary circulation, stronger midlevel inflow, larger $\theta_{e}$ in the sensitive region, and lower $\theta_{e}$ in the midlevel inflow region.

The correlations between other hydrometeors $\left(q_{c}, q_{r}, q_{i}\right)$ and TC intensification rate are generally smaller than $q_{v}$ (and $\theta_{e}$ ). 
(a)

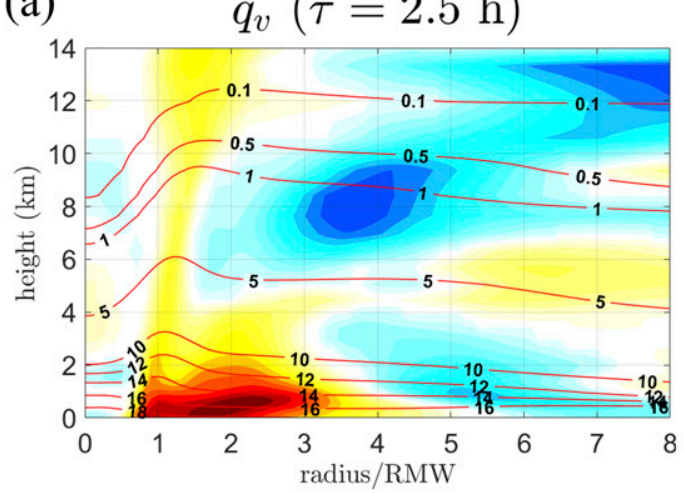

(c)

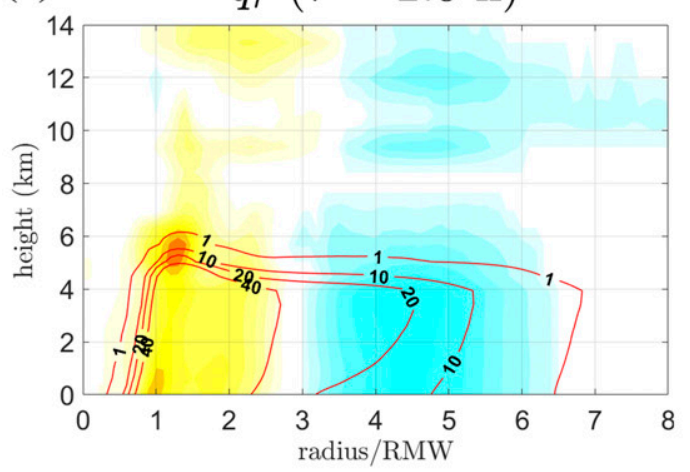

(e)

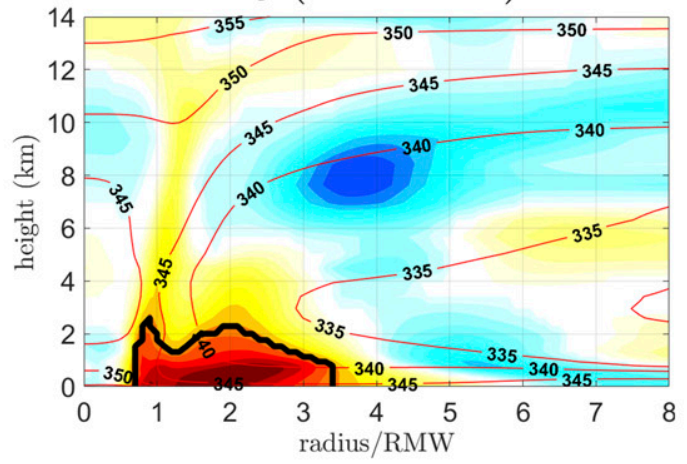

(b)

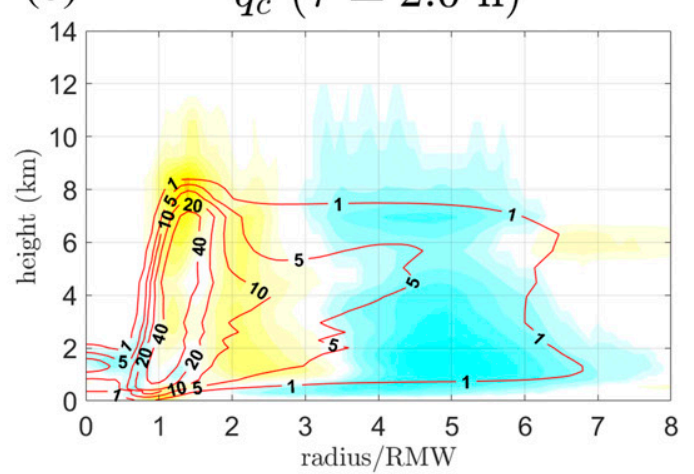

(d)

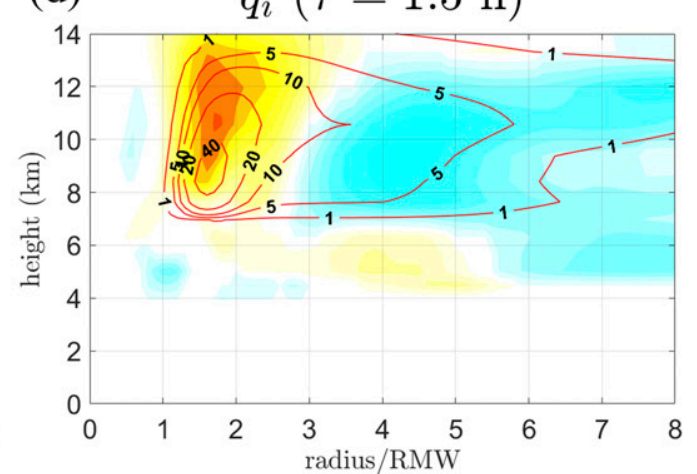

(f)

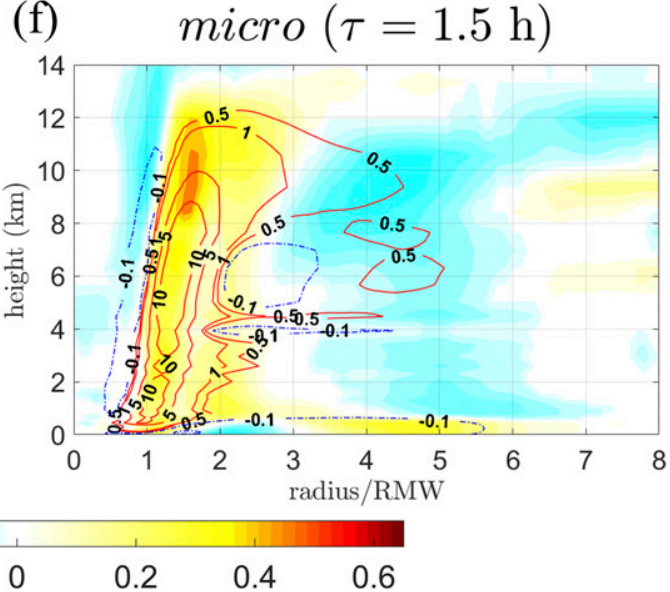

FIG. 6. As in Fig. 4, but for thermodynamic variables. (a) Water vapor mixing ratio $\left(\mathrm{g} \mathrm{kg}^{-1}\right)$. (b) Cloud water mixing ratio $\left(10^{-1} \mathrm{~g} \mathrm{~kg}^{-1}\right)$. (c) Rain water mixing ratio $\left(10^{-1} \mathrm{~g} \mathrm{~kg}^{-1}\right)$. (d) Ice mixing ratio $\left(10^{-1} \mathrm{~g} \mathrm{~kg}^{-1}\right)$. (e) Equivalent potential temperature $(\mathrm{K})$. (f) Microphysical latent heating $\left(10^{-3} \mathrm{~K} \mathrm{~s}^{-1}\right)$. The black contour in (e) is the sensitive region.

Figures $6 \mathrm{~b}-\mathrm{d}$ show that the amount of liquid and solid water substance in the interior (exterior) of the TC (here the boundary of interior and exterior is approximately 2.5 times the RMW) is positively (negatively) correlated with the intensification rate. It is noteworthy that $q_{i}$ (Fig. 6d) has larger correlations than $q_{c}$ (Fig. 6b) and $q_{r}$ (Fig. 6c). This is consistent with an observational study of $\mathrm{Wu}$ and Soden (2017), which suggested that strengthening storms have about $20 \%$-higher cloud ice water content than the weakening storms. The microphysical latent heating (Fig. 6f) shows a consistent pattern as the vertical wind (Fig. 4b) and $q_{i}$ (Fig. 6d) in the eyewall. It is noteworthy that the maximum positive correlation signal of both $q_{i}$ and microphysical latent heating occurs slightly inward and upward than the maximum of the mean field (Figs. 6d,f), implying a greater intensification rate if there is more heating located closer to the center.

Note that the correlations for the thermodynamic variables are generally greater than the dynamical variables, which is consistent with studies using the adjoint model (e.g., Ito et al. 2011; Doyle et al. 2012). This highlights the importance of thermodynamic variables in the intensification rate when we are certain about the 
(a)

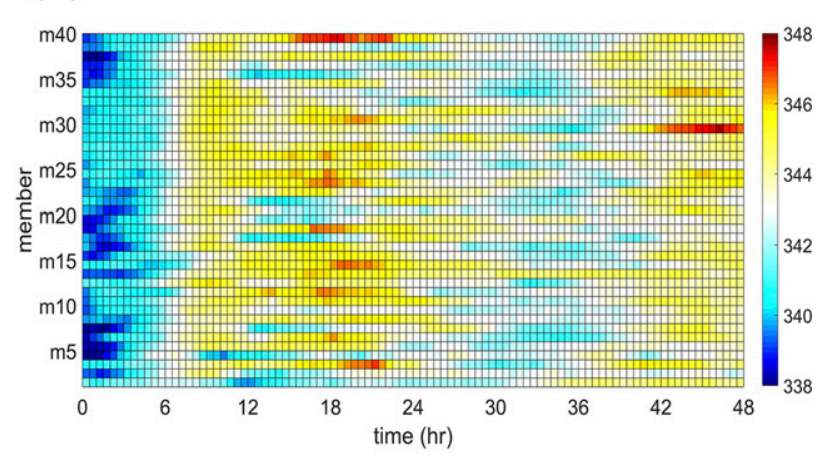

(b)

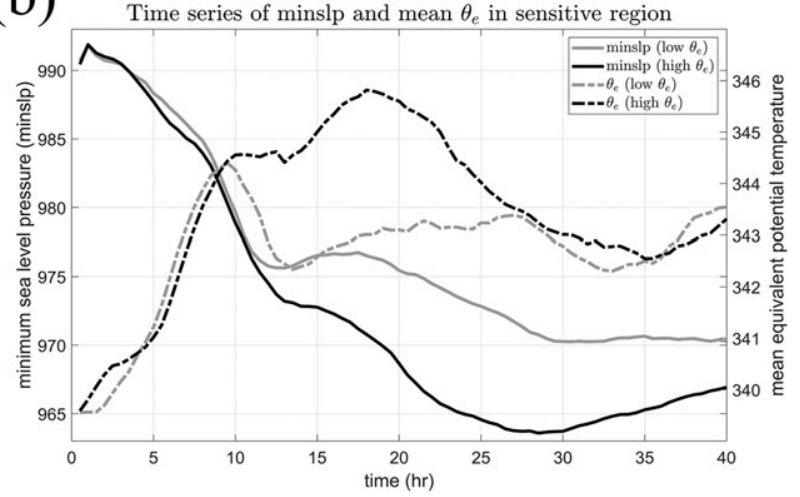

FIG. 7. (a) The mean equivalent potential temperature in the sensitive region at each time for each ensemble member. (b) The time series of the minimum sea level pressure (solid lines) and the mean equivalent potential temperature (dashed lines) in the sensitive region for the high $-\theta_{e}$ group (black lines) and low- $\theta_{e}$ group (gray lines).

initial intensity. The reason for the smaller correlation for dynamical variables may be related to the fact that the important dynamical variables for the intensification rate are closely related to the current intensity. When the current intensity is controlled, the uncertainty of these dynamical variables is also reduced.

\section{4. $\theta_{e}$ in the sensitive region}

The cause and effect of the $\theta_{e}$ variation in the sensitive region is examined in this section. Here, the formal definition of sensitive region is the region in which the partial correlation between $\theta_{e}$ and 2.5-h intensity change is larger than 0.45 . The black solid contour in Fig. 6e shows the sensitive region, and Fig. 7a shows the mean $\theta_{e}$ in the sensitive region at all times for each member. During $t=10-25 \mathrm{~h}$, there is a large variation of mean $\theta_{e}$ in the sensitive region; therefore we define this time period as the targeted time period. We partition our members into two groups with equal size: high $\theta_{e}$ and low $\theta_{e}$, according to their average $\theta_{e}$ in the sensitive region during the targeted time period.

Figure $7 \mathrm{~b}$ shows the time series of the group averaged intensity and group averaged mean $\theta_{e}$ in the sensitive region for each group. The time series of $\theta_{e}$ leads the time series of intensity change by $2.5-5 \mathrm{~h}$ in both groups. For example, the $\theta_{e}$ difference between the two groups begins at about $t=9 \mathrm{~h}$, while the intensity difference becomes apparent after $t=12 \mathrm{~h}$. This result demonstrates the high correlation between $\theta_{e}$ and the future intensification rate during the development stage of TC.

\section{a. Factors leading to different $\theta_{e}$ in the sensitive region}

To understand the factors leading to the different $\theta_{e}$ in the sensitive region for the high- and low- $\theta_{e}$ groups, we examine the Hovmöller diagram of radar reflectivity and $\theta_{e}$ averaged below $2 \mathrm{~km}$ (Fig. 8). Compared with the high $\theta_{e}$ group, it is found that the radar reflectivity at 2-5 times the RMW is stronger after $t=6 \mathrm{~h}$ for the low $-\theta_{e}$ group, which is related to the stronger outer rainband. The more active rainband in the low $-\theta_{e}$ group may be attributed to the larger $\theta_{e}$ beyond 2 times the RMW before $t=10 \mathrm{~h}$ (Fig. 8b). The $\theta_{e}$ inside the rainband begins to decrease $0.5-1 \mathrm{~h}$ after the development of the rainband, while the $\theta_{e}$ outside the rainband increases or remains stable with time for the low $\theta_{e}$ group (Fig. 8a). This suggests that there is a sharp difference of $\theta_{e}$ tendency radially inward and outward to the rainband. The decrease of $\theta_{e}$ inside the rainband for the low $-\theta_{e}$ group thus leads to the difference of $\theta_{e}$ from the high $-\theta_{e}$ group in the sensitive region after $t=9 \mathrm{~h}$ (Fig. $8 \mathrm{~b}$ ).

To quantitatively estimate how the rainband is associated with the decrease of $\theta_{e}$, we perform the budget analysis of $\theta_{e}$ in the region from the RMW to 3 times the RMW below $2 \mathrm{~km}$ (the sensitive region defined in the beginning of this section):

$$
\frac{\partial \theta_{e}}{\partial t}=-v_{r} \frac{\partial \theta_{e}}{\partial r}-w \frac{\partial \theta_{e}}{\partial z}+(\mathrm{HFX}+\mathrm{QFX})
$$

where HFX and QFX are the tendency from the surface sensible heat flux and moisture flux, respectively. The $\theta_{e}$ tendency and the advection terms are first calculated at each grid point, and then averaged in the sensitive region. ${ }^{8}$ Note that larger

${ }^{8}$ For HFX and QFX, we first calculate the area-integrated surface fluxes, and transform them into the tendency of the potential temperature $(\theta)$ and the water vapor mixing ratio $\left(q_{v}\right)$ by the following relations:

$$
\begin{aligned}
\frac{d q_{v}}{d t} & =\frac{\mathrm{QFX} \times \Delta A}{\mu_{d}}, \\
\frac{d \theta}{d t} \frac{C_{p} T_{v}}{\theta} & =\frac{\operatorname{HFX} \times \Delta A}{\mu_{d}},
\end{aligned}
$$

where $d q_{v} / d t$ and $d \theta / d t$ are the tendency of water vapor mixing ratio and potential temperature averaged in the sensitive region, $\Delta A$ is the surface area of the sensitive region, $\mu_{d}$ is the dry air mass in the sensitive region, and $T_{v}$ is the average virtual temperature in the sensitive region. We then calculate the average tendency of $\theta_{e}$ in the sensitive region based on the tendency of $q_{v}$ and $\theta$ :

$$
\begin{aligned}
\frac{d \theta_{e}}{d t} & =\frac{d \theta}{d t}\left(1+\frac{L_{v} q_{v}}{C_{p} T_{c}}\right)+\theta \frac{L_{v}}{T_{c} C_{p}}\left[\frac{d q_{v}}{d t}-\frac{q_{v}}{T_{c}} \frac{d T_{c}}{d t}\right] \\
& \approx \frac{d \theta}{d t}\left(1+\frac{L_{v} q_{v}}{C_{p} T_{c}}\right)+\frac{d q_{v}}{d t}\left(\frac{L_{v} \theta}{T_{c} C_{p}}\right),
\end{aligned}
$$

where $T_{c}$ is the condensation temperature. We have neglected the tendency of $T_{c}$ since $\left|\left(q_{v} / T_{c}\right)\left(d T_{c} / d t\right)\right| \ll\left|d q_{v} / d t\right|$. 
(a)

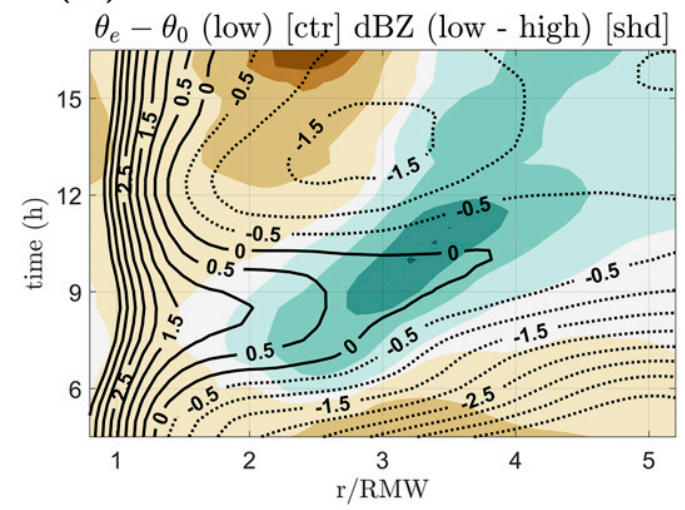

(b)

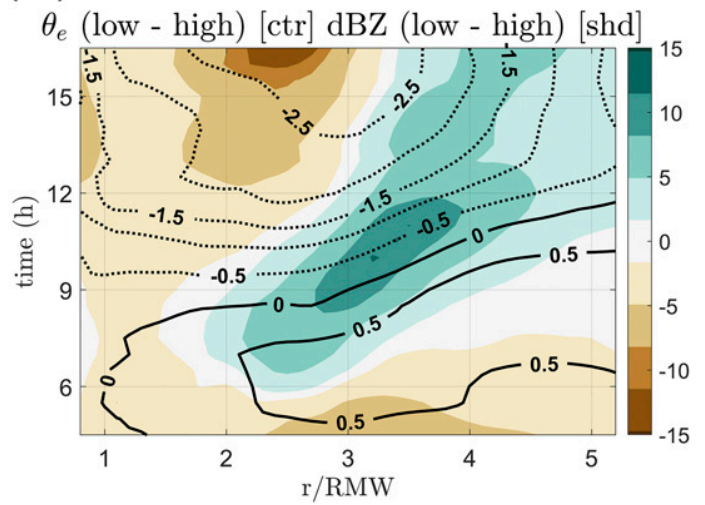

FIG. 8. (a) The Hovmöller diagram for the equivalent potential temperature averaged below $2 \mathrm{~km}$ for the low- $\theta_{e}$ group (contours, $\mathrm{K}$ ) and the difference of the radar reflectivity averaged below $2 \mathrm{~km}$ for the low- and high- $\theta_{e}$ group (shading, $\mathrm{dBZ}$ ). A reference temperature $\theta_{0}\left(\theta_{0}=342 \mathrm{~K}\right)$ is subtracted from the equivalent potential temperature for the low- $\theta_{e}$ group to emphasize the change of $\theta_{e}$. (b) The Hovmöller diagram for the difference of the equivalent potential temperature averaged below $2 \mathrm{~km}$ for the low- and high- $\theta_{e}$ groups (contours, $\mathrm{K}$ ). The shading is as in (a).

portion of the budget terms are contributed by the region closer to 3 times of RMW, since the area increases with radii. In addition, the sensitive region in the physical space can be slightly different with time (and member), while its effect on the budget analysis is negligible. The estimated $\theta_{e}$ tendency by the sum of the rhs of Eq. (5) is close to the actual $\theta_{e}$ tendency for both high- and low- $\theta_{e}$ groups (Fig. 9), although other effects (such as radiative cooling) are neglected.

By comparing the budget between the two groups during $t=$ 7.5-12 h, during which the rainband becomes stronger for the low- $\theta_{e}$ group (Fig. 8), we find that the variation of radial advection (ADVR) is mainly responsible for the variation of $\theta_{e}$ tendency during this time (Fig. 9c). Note that the negative ADVR is due to the negative gradient of $\theta_{e}$ in the sensitive region. This suggests that the cold pool generated by the rainband is advected inward to decrease the $\theta_{e}$ in the sensitive region for the low- $\theta_{e}$ group.

\section{b. TC response to different $\theta_{e}$ in the sensitive region}

The dynamical processes associated with the larger $\theta_{e}$ in the sensitive region is examined in this section. The increase of $\theta_{e}$ in the sensitive region is associated with the decrease of convective stability (the vertical derivative of $\theta_{e}$ ) in the lower eyewall at about $3 \mathrm{~km}$. Figure 10 shows that the convective stability from RMW to 2 times the RMW at $3-4 \mathrm{~km}$ is lower in the high- $\theta_{e}$ group, and becomes even lower during $t=13-18 \mathrm{~h}$. The larger instability during the parcel's ascent heats the parcel more, leading to a stronger updraft (gray dashed lines in Fig. 10a).

In addition to the strength of the vertical motion in the eyewall, its radial location in mid- to upper levels is also important to TC intensification. This is because the vertical motion in midto upper levels is closely related to the heating, and the heating efficiency can be higher if the heating is closer to the TC center (Schubert and Hack 1982; Vigh and Schubert 2009; Rogers et al. 2013; Wang and Wang 2014; Chang and Wu 2017). It is found that the updraft in the high- $\theta_{e}$ group becomes steeper in slope, leading to an inward shift of vertical motion in mid- to upper level (Fig. 11a). The updraft in the low $\theta_{e}$ group also becomes slightly stronger, while the radial location of the vertical motion in mid- to upper levels does not change (Fig. 11b).

To identify factors leading to the different radial locations of the vertical motion in mid- to upper levels, we examine the horizontal convergence in the boundary layer. It is found that the location of the horizontal convergence in the boundary layer remains similar for both groups (Figs. 11a,b). In addition, the changes of boundary layer convergence for both groups are similar during $t=13-18 \mathrm{~h}$ (Figs. 11c,d). This suggests that changes in the boundary layer convergence are not the primary reason for the changes in vertical motion in the mid- to upper levels.

The main difference between the two groups in the change of horizontal convergence during $t=13-18 \mathrm{~h}$ can be identified in the region above $3 \mathrm{~km}$ (Figs. 11c,d). With the larger convective instability above $3 \mathrm{~km}$ for the high- $\theta_{e}$ group, there is an enhanced vertical acceleration given by the increased latent heat release. Based on mass conservation, the enhanced vertical acceleration can be inferred from the stronger horizontal convergence above $3 \mathrm{~km}$ (Fig. 11c). The enhanced vertical acceleration causes a more upright updraft, and thereby an inward shift of vertical motion in mid- to upper levels. Therefore, it is suggested that the enhanced vertical acceleration above $3 \mathrm{~km}$ due to the release of convective instability is the primary factor for the different slope of updraft between the two groups.

The different distribution of vertical wind in the eyewall can redistribute the absolute angular momentum (AAM). We focus our discussions on the AAM change in the mid- to upper eyewall (between RMW to 2 times the RMW at 4-12 km). Since the effect of friction is negligible above the boundary layer, the change of AAM is almost only affected by the advection. It is found that both the change and the mean of vertical advection are almost balanced by the radial advection for both groups (Figs. 12a,b,d,e). This is because when the parcels from the lower eyewall brings high AAM $\left(M_{0}\right)$ to the 

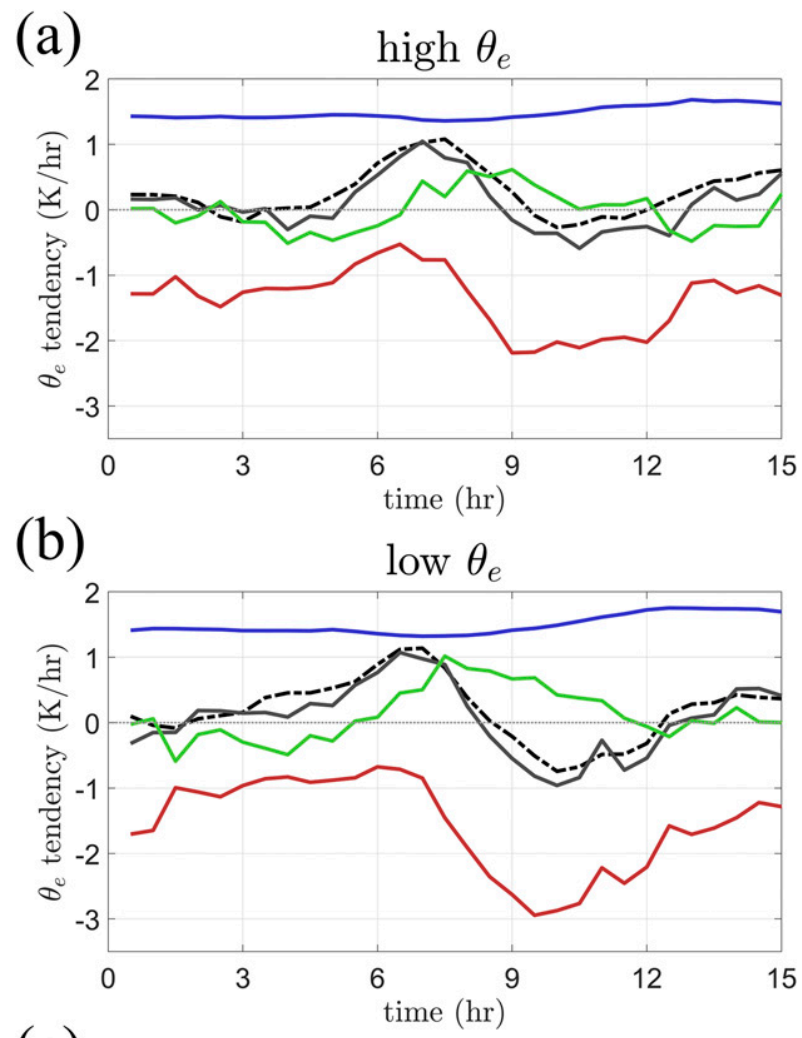

(c)

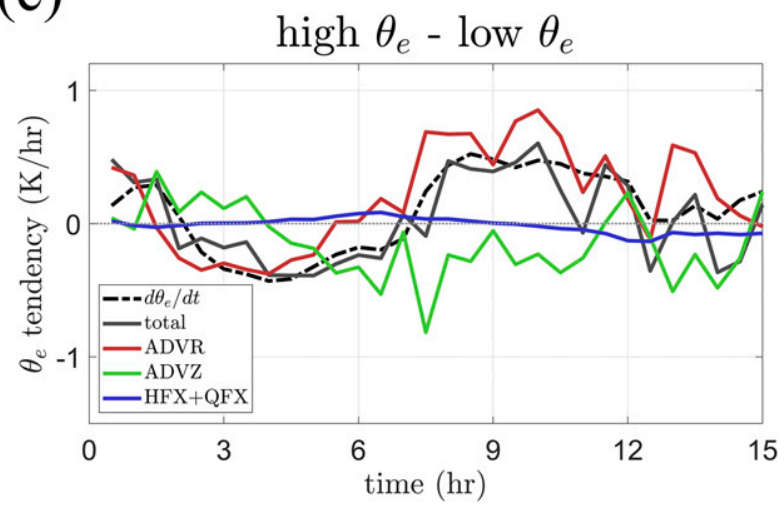

FIG. 9. The equivalent potential temperature budget averaged in the region from the RMW to 3 times the RMW below $2 \mathrm{~km}$. The black dashed line is the actual equivalent potential temperature tendency $\left(\mathrm{K} \mathrm{h}^{-1}\right)$, the black line is the sum of all the terms on the right-hand side of Eq. (5) $\left(\mathrm{K} \mathrm{h}^{-1}\right)$, the red line is the radial advection (ADVR) term $\left(\mathrm{K} \mathrm{h}^{-1}\right)$, the green line is the vertical advection (ADVZ) term $\left(\mathrm{K} \mathrm{h}^{-1}\right)$, and the blue line is the tendency from the surface fluxes $\left(\mathrm{K} \mathrm{h}^{-1}\right)$, including the sensible heat (HFX) and moisture (QFX) flux, for the (a) high- and (b) low- $\theta_{e}$ groups (c) and the differences between (a) and (b).

region with lower AAM $\left(M_{0}-\delta M\right)$, the radial force tends to push the parcels outward back to the original AAM surface $\left(M_{0}\right)$ as it is inertially stable in the eyewall ( $\left.\partial \mathrm{AAM} / \partial r>0\right)$. However, since the outward acceleration is not pushing the parcels back to the original AAM surface $\left(M_{0}\right)$ immediately, the total tendency of AAM is still positive. Therefore, with enhanced vertical acceleration in the high- $\theta_{e}$ group (Fig. 11c), the total AAM tendency is expected to be a small positive term from the imbalance between positive vertical advection and negative radial advection. Specifically, the vertical advection is estimated to be $80 \mathrm{~m}^{2} \mathrm{~s}^{-2}$ in the mid- to upper eyewall (Fig. 12a), and the tendency there is estimated to be only $2.8 \mathrm{~m}^{2} \mathrm{~s}^{-2}$ (Fig. 12c). Comparison of the AAM changes for both groups shows that the high- $\theta_{e}$ group has a positive change of AAM in the mid- to upper eyewall (Fig. 12c), while that change of AAM for the low- $\theta_{e}$ group is much smaller (Fig. 12f) due to the weaker vertical acceleration.

The redistribution of AAM can then affect the inertial stability $\left(I^{2}\right)$, which is

$$
I^{2}=\frac{1}{r^{3}} \frac{\partial(A A M)^{2}}{\partial r} .
$$

For the high $-\theta_{e}$ group, the increase of AAM in the eyewall increases the gradient of AAM inside of the eyewall. Since AAM monotonically increases with radius near the eyewall, the increase of AAM gradient therefore increases the inertial stability inside the eyewall (Fig. 12c). The increase of inertial stability has been shown to enhance the heating efficiency, which is favorable for TC intensification (e.g., Schubert and Hack 1982; Vigh and Schubert 2009). For the low- $\theta_{e}$ group, the increase of AAM and inertial stability in the eyewall are both less apparent (Fig. 12f).

Based on Figs. 10-12, a mechanism for increased $\theta_{e}$ leading to greater TC intensification rate is summarized as follows. As $\theta_{e}$ becomes larger in the sensitive region, $\theta_{e}$ above the boundary layer (around $3 \mathrm{~km}$ ) becomes larger due to the vertical advection. Higher $\theta_{e}$ decreases the convective stability, causing the parcel to undergo enhanced vertical acceleration, which leads to a more upright updraft, and an inward shift of vertical motion and heating in the mid- to upper eyewall. The upright trajectory of the parcels with enhanced vertical acceleration is more likely to increase AAM in the eyewall by a small yet positive amount, as a result of the small imbalance between vertical and radial advections. The increase of AAM in the eyewall leads to an increase of $I^{2}$ inside the eyewall. The larger heating and the inward location of heating together lead to the greater intensification rate. Although larger heating associated with higher $\theta_{e}$ in the sensitive region is well known, one important finding highlighted here is that the location of heating in the mid- to upper eyewall is also influenced by the $\theta_{e}$ in the sensitive region.

\section{c. The role of outer rainband in $\theta_{e}$ in the sensitive region}

We have focused our discussion on the difference between the high- and low- $\theta_{e}$ groups before $t=18 \mathrm{~h}$ in the previous subsections. After $t=18 \mathrm{~h}$, examinations of the Hovmöller diagram of radar reflectivity and $\theta_{e}$ for all the members indicate that the outer rainband development is closely related to the $\theta_{e}$ in the sensitive region (figures not shown). However, since the time for the rainband development for each of the member is different, we choose the 40th member, which has a clear rainband development period, as an example to demonstrate the relation between the rainband and $\theta_{e}$. 

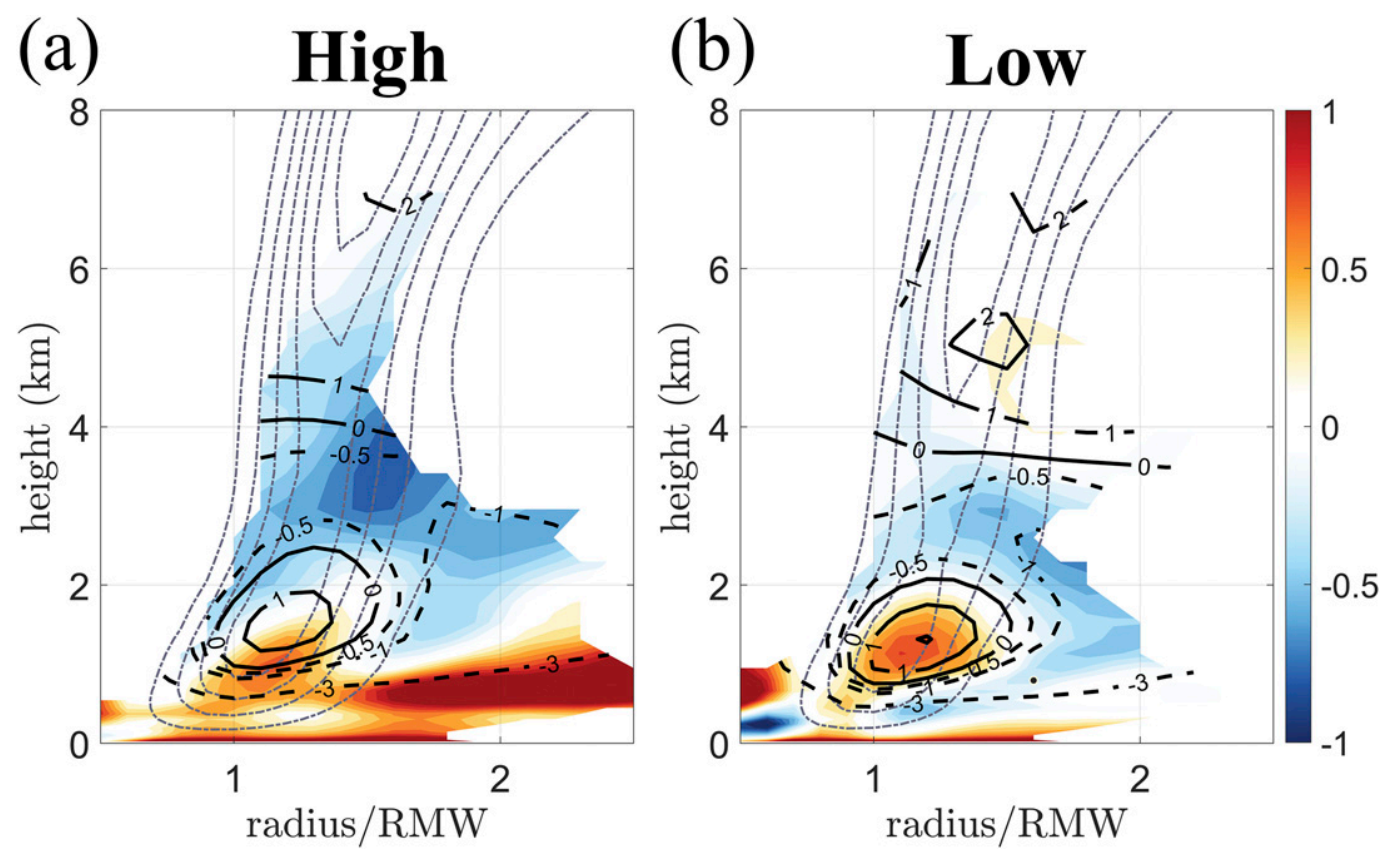

FIG. 10. The mean convective stability (contoured in black, $\mathrm{K} \mathrm{km}^{-1}$ ), the vertical motion (contoured in gray, with a contour interval of $0.5 \mathrm{~m} \mathrm{~s}^{-1}$ ), and the change (shaded, $\mathrm{K} \mathrm{km}^{-1}$ ) of convective stability during $t=13-18 \mathrm{~h}$ for the (a) high- and (b) low- $\theta_{e}$ groups. For the convective stability and the change of convective stability, only the region with the relative humidity greater than $90 \%$ is shown. The $x$ axis is the radius normalized by RMW and the $y$ axis is the height.

Some features of rainband development can be identified during $t=18-36 \mathrm{~h}$ in Fig. 13. There is an upward (downward) motion in the leading (rear) side of the outward propagating positive radar reflectivity during this period (Fig. 13a). The midlevel inflow is found to be stronger from $t=15-21 \mathrm{~h}$ during the initiation of a rainband, and the low-level inflow is stronger as well (Figs. 13b,c). This is consistent with the finding that the stronger midlevel inflow is associated with the stronger secondary circulation (Figs. 5a,b). After $t=30 \mathrm{~h}$, the low-level inflow and $\theta_{e}$ are reduced near 2-5 times the RMW in the rear of the rainband (Figs. 13b,d), which is consistent with the features of rainbands identified in Kimball (2006).

To see how the reduction of $\theta_{e}$ in the sensitive region is associated with the outer rainband, parcel trajectories reaching the sensitive region are examined. The read/interpolate/plot $(\mathrm{RIP})^{9}$ is utilized to perform the backward and forward trajectory calculation. ${ }^{10}$ The trajectories of parcels at two time steps are selected: one is the time at the initiation of a rainband ( $t=20 \mathrm{~h}$, abbreviated as t20) during which $\theta_{e}$ in the sensitive region is high, and the other is the time when the rainband is well developed ( $t=32 \mathrm{~h}$, abbreviated as $\mathrm{t} 32$ ) during which the $\theta_{e}$ in the sensitive region becomes lower. Analysis is performed on trajectories for $12 \mathrm{~h}$ before and after $t$ (each referred to as forward and backward trajectories).

\footnotetext{
${ }^{9}$ See http://www.mmm.ucar.edu/wrf/users/docs/ripug.htm.

${ }^{10}$ Model data are interpolated from 30 min per output into 6 min per output when performing the trajectory analysis.
}

It is found from the backward trajectory analysis that the origin of parcels can be generally divided into two regions: one from the midlevel, and the other from the boundary layer (Figs. 14a,c). The parcels are therefore partitioned into two groups according to their altitude at $12 \mathrm{~h}$ before the start of trajectory analysis: ${ }^{11}$ parcels below $2 \mathrm{~km}$ are classified as parcels from the boundary layer, while those at and above $2 \mathrm{~km}$ are defined as coming from the midlevel. It is found that $80.0 \%$ and $71.5 \%$ of the parcels in the sensitive region are from the boundary layer for $\mathrm{t} 20$ and $\mathrm{t} 32$, respectively. This result suggests that the $\theta_{e}$ in the boundary inflow layer is an important factor controlling the $\theta_{e}$ in the sensitive region, which is consistent with the result from the $\theta_{e}$ budget that the radial advection is dominant. For the parcels from the midlevel, t32 has a larger portion $(28.5 \%)$, which can be explained by the stronger midlevel inflow around $t=20 \mathrm{~h}$ (Fig. 13c). The larger portion of parcels from the midlevel can result in the lower $\theta_{e}$ in the sensitive region for $\mathrm{t} 32$.

In addition to the different origins of parcels, the variation of $\theta_{e}$ in the boundary inflow layer can also affect $\theta_{e}$ in the sensitive region. For $\mathrm{t} 20$, the $\theta_{e}$ of parcels from the boundary layer gradually increases (Fig. 15a) before reaching the sensitive region $(0 \mathrm{~h})$, while that of $\mathrm{t} 32$ remains steady (Fig. 15b). The surface heat flux for $\mathrm{t} 32$ is actually larger than that in $\mathrm{t} 20$ due to the larger air-sea contrast and the expansion of surface wind

\footnotetext{
${ }^{11}$ For analysis starting at $t=20,-12 \mathrm{~h}$ corresponds to $t=8 \mathrm{~h}$; for analysis starting at $t=32,-12 \mathrm{~h}$ corresponds to $t=20 \mathrm{~h}$.
} 

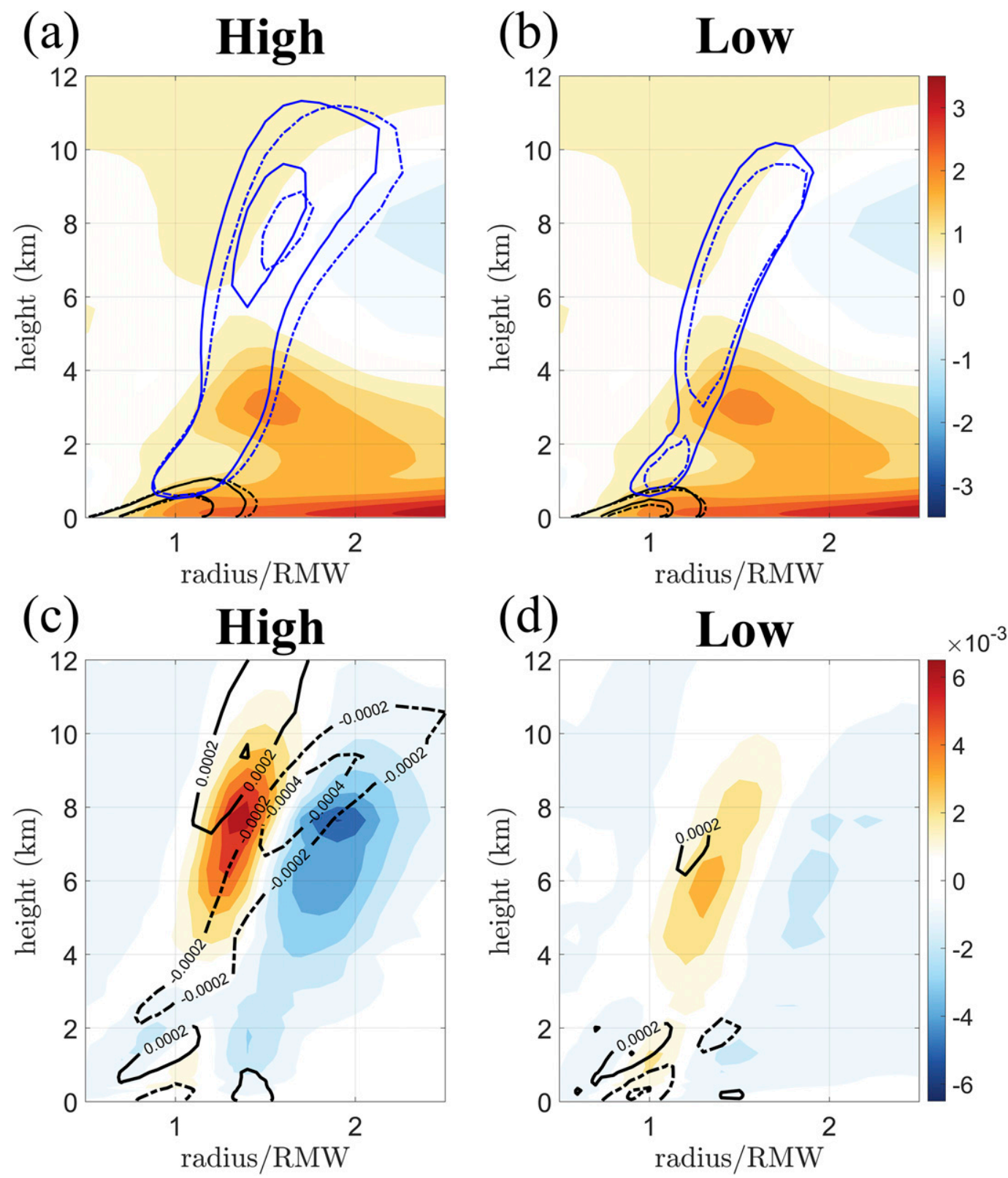

FIG. 11. (a),(b) The mean radial convergence (only the region below $2 \mathrm{~km}$ is shown) during $t=13-15 \mathrm{~h}$ (dashed black contours, with a contour interval of $10^{-3} \mathrm{~s}^{-1}$ ), during $t=16-18 \mathrm{~h}$ (solid black contours, with a contour interval of $10^{-3} \mathrm{~s}^{-1}$ ), and the mean vertical motion during $t=13-15$ (dashed blue contours, with a contour interval of $1.5 \mathrm{~m} \mathrm{~s}^{-1}$ ), and during $t=16-18 \mathrm{~h}$ (solid blue contours, with a contour interval of $1.5 \mathrm{~m} \mathrm{~s}^{-1}$ ) for (a) high-and (b) low- $\theta_{e}$ groups. The shading in both panels is the difference (high - low) of the mean equivalent potential temperature (K) during $t=13-18 \mathrm{~h}$ for the high- and low- $\theta_{e}$ groups. (c),(d) The change of radial convergence (contours, with $z$ contour interval $2 \times 10^{-4} \mathrm{~s}^{-1}$ ) and the change of microphysical heating (shading, $\mathrm{K} \mathrm{s}^{-1}$ ) during $t=13-18 \mathrm{~h}$ for the (c) high- and (d) low- $\theta_{e}$ groups. The $x$ axis is the radius normalized by RMW and the $y$ axis is the height.

associated with the rainband (figures not shown). For this reason, it is suggested that mixing with the low $-\theta_{e}$ air caused by the rainband can play a substantial role in limiting the increase of $\theta_{e}$ in the boundary layer, and therefore $\theta_{e}$ cannot be immediately recovered by surface fluxes for $\mathrm{t} 32$. This is consistent with the findings in Alland et al. (2017).
For the forward trajectory analysis, differences occur between t20 and t32 after $1 \mathrm{~h}$ (figures not shown) and persist for the following hours. For $\mathrm{t} 20$ (with higher $\theta_{e}$ ), a large portion of the parcels are lifted to mid- to upper troposphere (Fig. 14b), while most of the air parcels stay in their original place for $\mathrm{t} 32$ (Fig. 14d). Furthermore, the signal of outer rainband development can be 
(a) High (ADVZ)

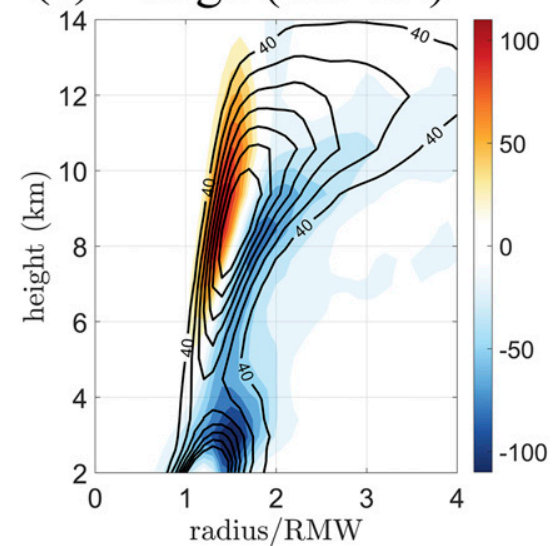

(d) Low (ADVZ)

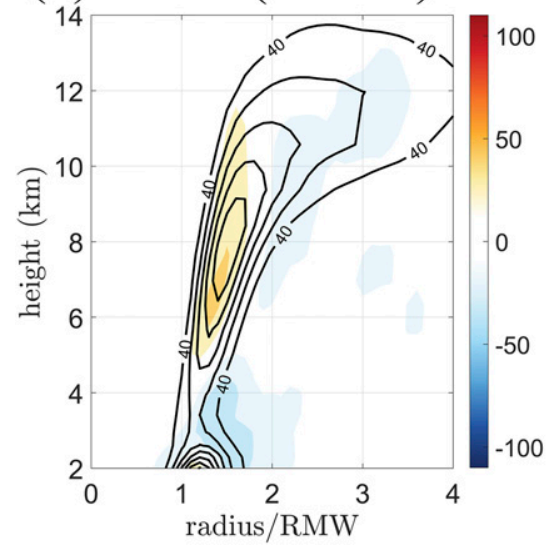

(b) High (ADVR)

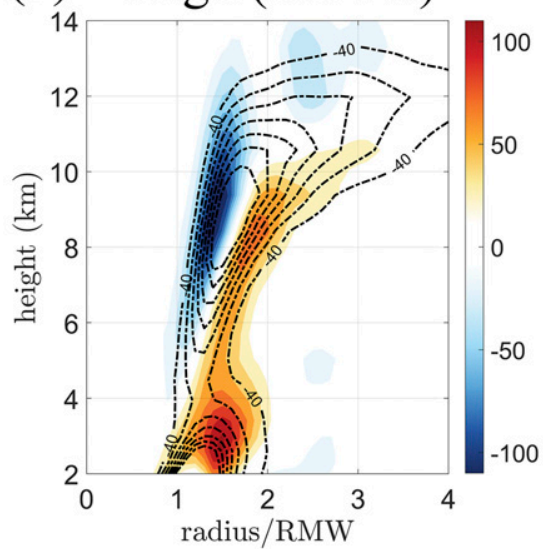

(e) Low (ADVR)

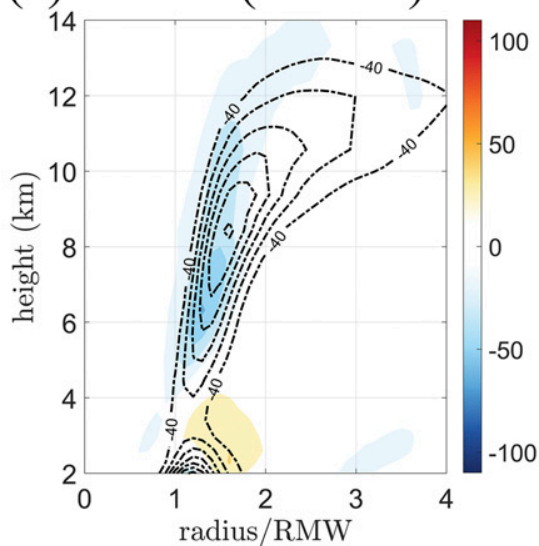

(c) High (TEND)

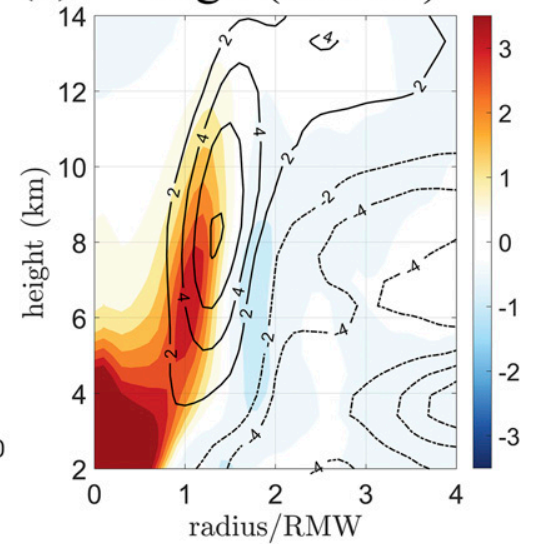

(f) Low (TEND)

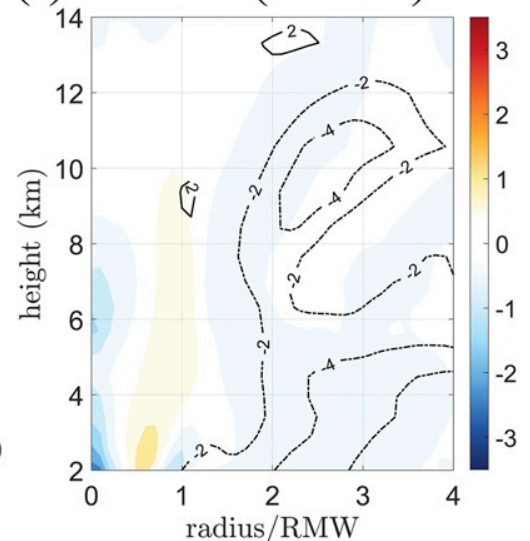

FIG. 12. The mean (black contours, with a contour interval of $40 \mathrm{~m}^{2} \mathrm{~s}^{-1}$ ) and the change (shaded, $\mathrm{m}^{2} \mathrm{~s}^{-1}$ ) of the (a),(d) vertical and (b),(e) radial advection of the absolute angular momentum during $t=13-18 \mathrm{~h}$. (c),(f) The change of absolute angular momentum (contours, $10^{4} \mathrm{~m}^{2} \mathrm{~s}^{-1}$ ) and inertial stability (shaded, $10^{-6} \mathrm{~s}^{-2}$ ) during $t=13-18 \mathrm{~h}$. (a)-(c) The high- $\theta_{e}$ group and (d)-(f) the low- $\theta_{e}$ group. The $x$ axis is the radius normalized by RMW and the $y$ axis is the height.

found in Fig. 14b. For the forward trajectory, both the $\theta_{e}$ in t20 and $\mathrm{t} 32$ decreases during $0-2 \mathrm{~h}$, which is due to the mixing with lower environmental $\theta_{e}$. In particular, the variance of $\theta_{e}$ in $\mathrm{t} 32$ almost vanishes because most of the parcels stay in the same place and mixing appears evident (Fig. 15b).

Several issues regarding to the midlevel inflow merit future investigations. First, its origin is still uncertain. Corsaro and Toumi (2017) showed that air parcels expelled from the deep convection into the outflow layer can recirculate back into the vortex and weaken the TC on a beta plane. Similarly, our results show that with the same TC intensity, when the secondary circulation is stronger, which implies a period of active convection, the midlevel inflow also becomes stronger. However, our simulations are on an $f$ plane and our midlevel inflow seems to take place earlier than that in Corsaro and Toumi's (2017) simulations. Therefore, it would be interesting to know whether the recirculation of the outflow from the deep convection is the main cause of the stronger midlevel inflow.

Second, how the midlevel inflow affect the TC structure is another open question. Since the midlevel inflow brings the low- $\theta_{e}$ and high-density air to the surface, which may create a region of negative density gradient and then act as a dynamical driving force for the initiation of convection. Furthermore, the active convection can sometimes lead to the secondary eyewall formation. Some recent studies have shown that the descending midlevel inflow can be a pathway for the secondary eyewall formation (Yu and Didlake 2019).

Last but not least, the effect of midlevel inflow on TC intensification can depend on the sea surface temperature (SST). Since the SST is fixed at $27^{\circ} \mathrm{C}$ in this study, the surface flux may not be large enough to immediately recover the reduced $\theta_{e}$ due to the entrainment of the midlevel inflow or the rainband. However, if the SST is higher, the recovery of $\theta_{e}$ can be faster, which may make the TC intensification rate less sensitive to the midlevel inflow or the rainband.

\section{Verification experiments}

A set of experiments are conducted to verify the statement from the partial correlation analysis that $q_{v}$ (or $\theta_{e}$ ) is positively (negatively) correlated with TC intensification rate inside 


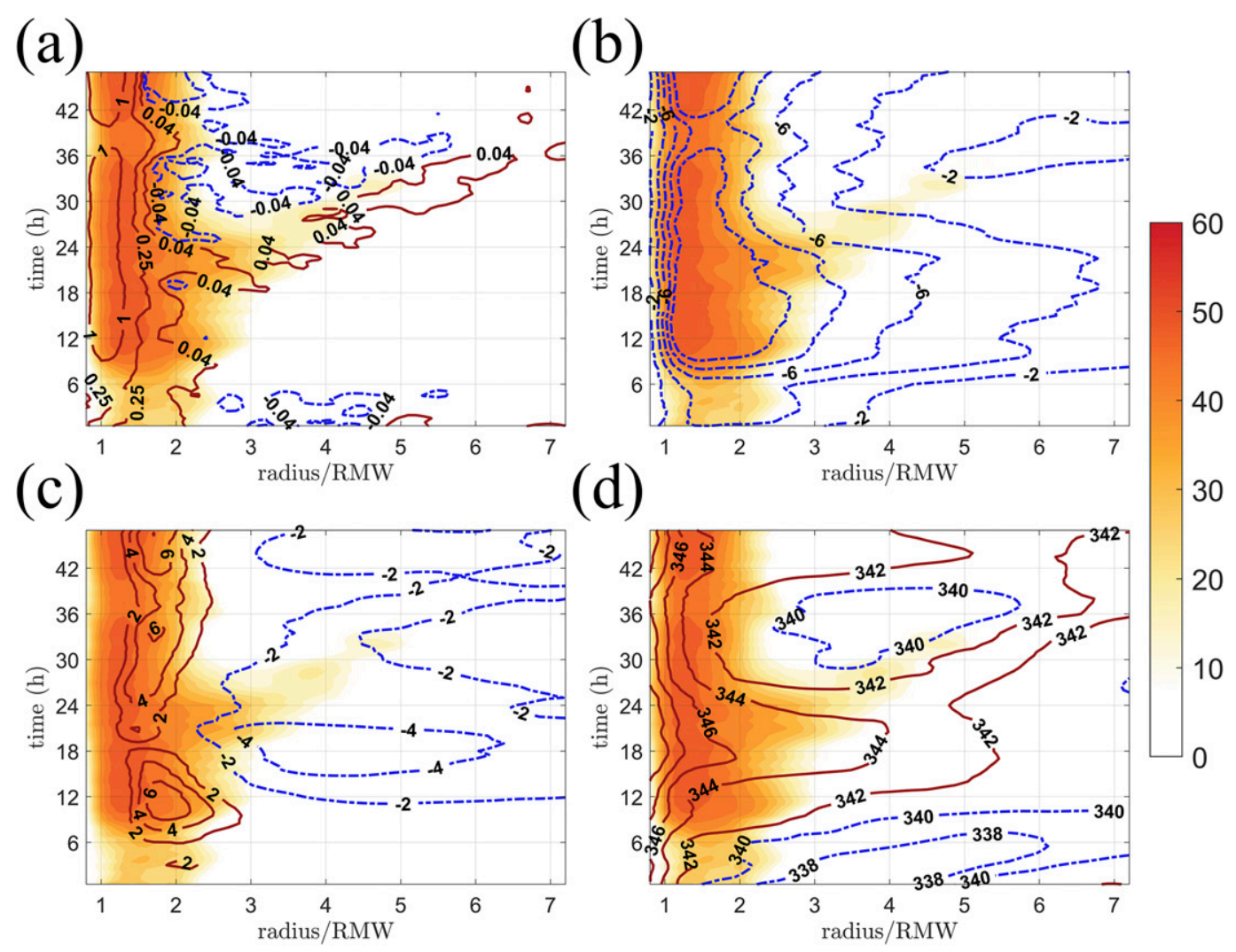

FIG. 13. The Hovmöller diagram for several variables of the 40th member. The shading in all panels is the radar reflectivity averaged below $2 \mathrm{~km}\left(\mathrm{dBZ}\right.$ ). (a) Vertical motion averaged below $2 \mathrm{~km}$ (contours, $\mathrm{m} \mathrm{s}^{-1}$ ). (b) Radial wind averaged below $2 \mathrm{~km}$ (contours, $\mathrm{m} \mathrm{s}^{-1}$ ). (c) Radial wind averaged between 7 and $10.5 \mathrm{~km}$ (contours, $\mathrm{m} \mathrm{s}^{-1}$ ). (d) Equivalent potential temperature averaged below $2 \mathrm{~km}$ (contours, $\mathrm{K}$ ).

(outside) the region of 5 times the RMW. The $q_{v}$ perturbations at different locations in the boundary layer are added to the 48-h output from CTRL run (the same time for the initiation of the ensemble). Specifically, the $q_{v}$ perturbations at radius $j$ times the RMW $\left(q_{v, j}^{\prime}\right)$ is set as

$$
\begin{aligned}
& q_{v, j}^{\prime}(\tilde{r}, z)=\min \left[ \pm 0.1 q_{v}(\tilde{r}, z) \hat{w}_{j}(\tilde{r}, z), q_{v s}(\tilde{r}, z)-q_{v}(\tilde{r}, z)\right], \\
& \hat{w}_{j}(\tilde{r}, z)=\frac{\tanh \left[-3\left(z-z_{0}\right)+1\right]}{2} \exp \left[-\left(\tilde{r}-r_{j}\right)^{2}\right]
\end{aligned}
$$

where $\hat{w}_{j}$ is a weighting function determining the shape and location of the perturbation, $z_{0}$ is a parameter for the depth of perturbation $\left(z_{0}=2 \mathrm{~km}\right), r_{j}$ is the center location of the perturbation $\left(r_{j}=1,2, \ldots, 8 \mathrm{RMW}\right)$, and $q_{v s}$ is the saturation $q_{v}$. An example of the weighting function centered at 3 times the RMW $\left(\hat{w}_{3}\right)$ is shown in Fig. 16a. The $q_{v}$ perturbations are $10 \%$ more (less) than the original $q_{v}$ in the area masked by the weighting function. An example with positive $10 \% q_{v, 3}^{\prime}$ centered at 3 times the RMW (abbreviated as $P_{3}$ ) is shown in Fig. 16b. A list of descriptions for all the verification experiments is shown in Table 1.

To quantify the sensitivity of TC intensification rate to $q_{v}$ at different locations, we calculate how much change of the average
mSLP during $t=0-5 \mathrm{~h}$ is given by the average change ${ }^{12}$ of $1 \mathrm{~g} \mathrm{~kg}^{-1} q_{v}$ at different radius, which is shown in Eq. (9):

$$
\begin{aligned}
& \text { sensitivity }_{\text {experiment(I)-experiment(II) }} \\
& =\frac{-\left(\mathrm{mSLP}_{\text {experiment(I) }}-\mathrm{mSLP}_{\text {experiment(II) }}\right)}{q_{v \text { experiment(I) }}-q_{v \text { experiment(II) }}} .
\end{aligned}
$$

Three kinds of differences can be used to estimate the sensitivity: 1) experiments with positive perturbations and CTRL, 2) experiments with negative perturbations and CTRL, and 3) experiments with positive perturbations and experiments with negative perturbations. Note that if the response of TC intensity is linearly related to the $q_{v}$ at a certain radius, the sensitivity estimated by these three different ways (positive - CTRL, negative - CTRL and positive - negative) should be exactly the same. Figure 17 shows that although there are some differences of the sensitivity at a fixed radii between experiments (blue, red

\footnotetext{
${ }^{12}$ The average change of $q_{v}$ at radius $j$ RMW is given by the average $q_{v}$ perturbation in the volume between $(j-0.5) \mathrm{RMW}$ and $(j+0.5)$ RMW below $1.5 \mathrm{~km}$.
} 
(a) $\quad$ t20 $(-12 h, t=8 h)$

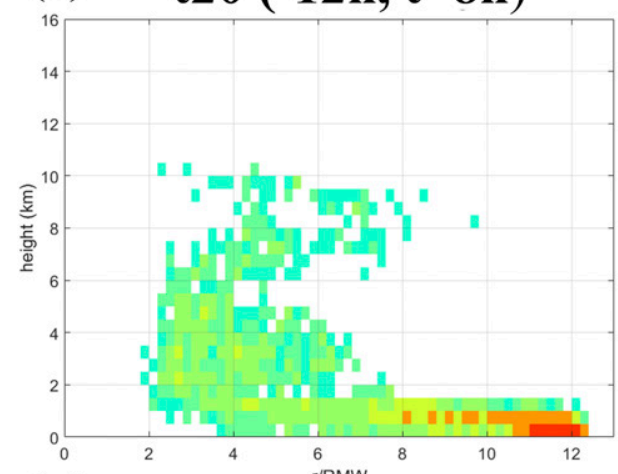

(c) $\mathbf{t 3 2}(\mathbf{- 1 2 h}, \mathbf{t}=\mathbf{2 0 h})$

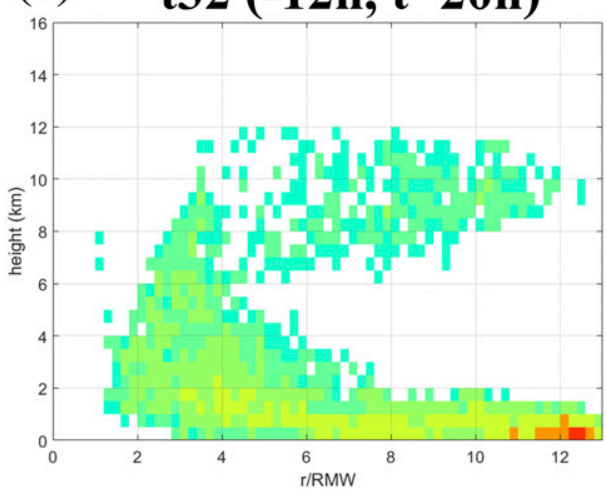

(b) $\mathbf{t 2 0}(+12 \mathrm{~h}, \mathrm{t}=\mathbf{3 2 h})$

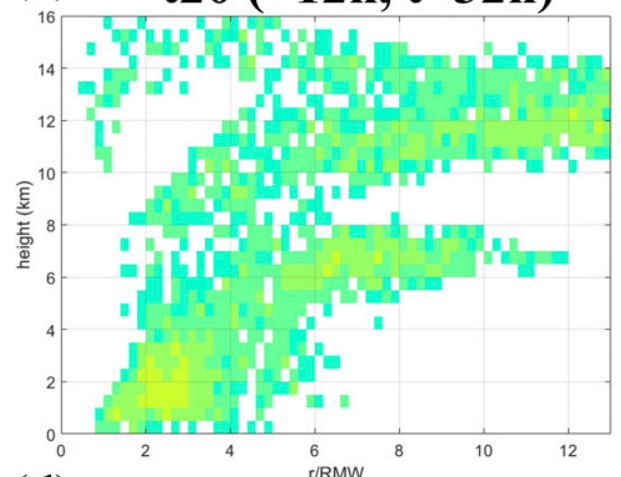

(d) $\mathbf{t 3 2}(+\mathbf{1 2 h}, \mathbf{t}=\mathbf{4 4 h})$

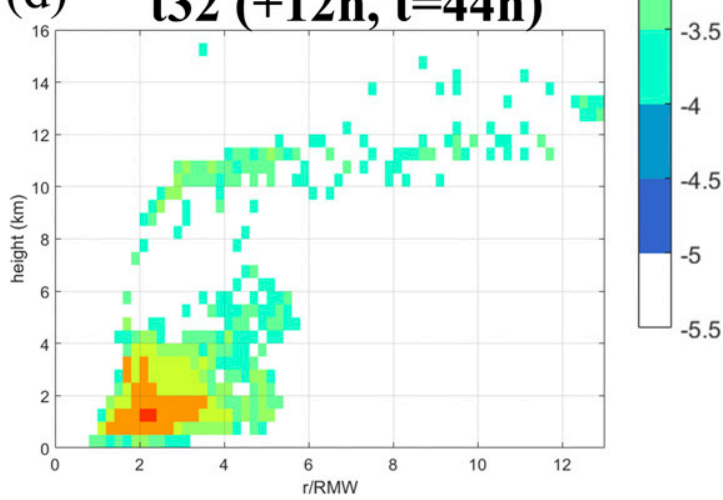

FIG. 14. The logarithm of the probability density function of parcel distribution at $12 \mathrm{~h} \mathrm{(a),(c)} \mathrm{before} \mathrm{and} \mathrm{(b),(d)}$ after the release of parcels in the 40th member. The time for the release of parcels is $t=20 \mathrm{~h}$ and $t=32 \mathrm{~h}$ for t 20 and $\mathrm{t} 32$, respectively. The first number in the parenthesis is the time relative to the release of parcels, and second number is the absolute time.

stars and the bar), their differences are not too large and at least they are qualitatively consistent. It is interesting to note that at around 3-4 times the RMW, the sensitivity are also quantitatively similar, suggesting a linear response of TC intensification rate to $q_{v}$ (within $10 \%$ of the perturbations) in these regions.
Figure 17 also shows that the sensitivity is largest within 2 times the RMW, with about $2-3 \mathrm{hPa}$ decrease (increase) in mSLP for $1 \mathrm{~g} \mathrm{~kg}^{-1}$ increase (decrease) of $q_{v}$ at the RMW or at the 2 times the RMW. This change of $q_{v}$ is about $5 \%-6 \%$. In addition, the sensitivity is positive (negative) inside (outside) (a)

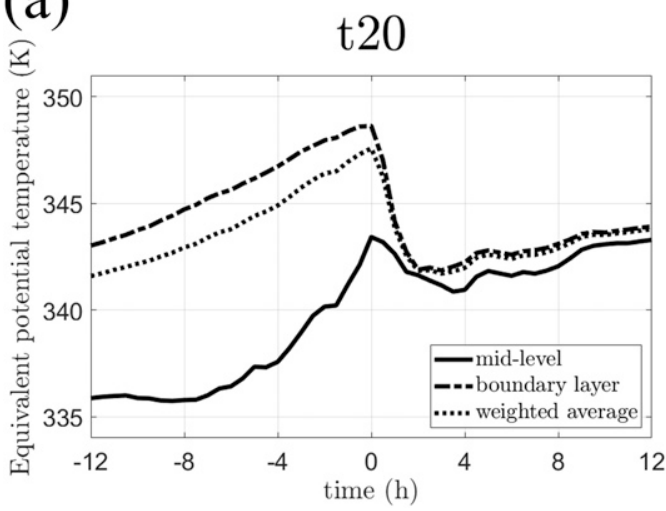

(b)

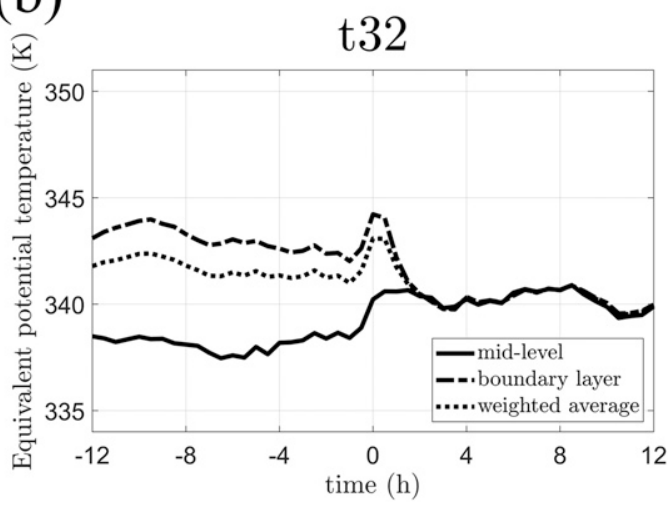

FIG. 15. The time evolution of the average equivalent potential temperature of parcels originated from the boundary layer (dash-dotted line), midlevel (solid line), and their weighted average (dotted line) for (a) t20 and (b) t32. 
(a)

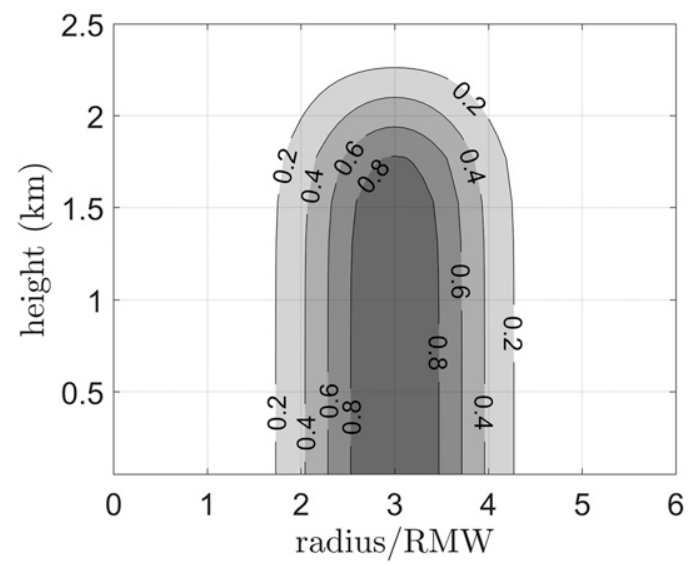

(b)

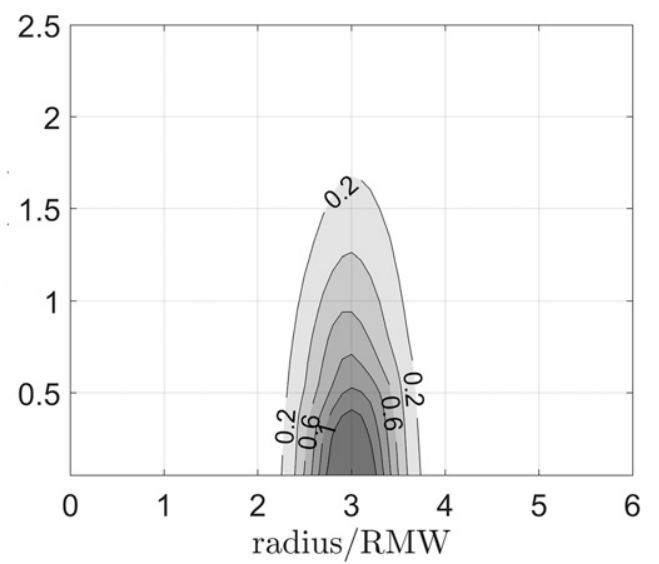

FIG. 16. (a) The weighting function $\hat{w}_{3}$. (b) The water vapor perturbation for the verification experiment $P_{3}\left(\mathrm{~g} \mathrm{~kg}^{-1}\right)$.

5 times the RMW. These results are consistent with the partial correlation results in section 3 .

The negative impact of outer-region moisture in the TC intensification rate has been found to be closely related to the outer rainband. The larger moisture in the outer region enhances the outer rainband, which reduces the intensification rate both by bringing low- $\theta_{e}$ air into the boundary layer and through reduction of the low-level radial inflow into the eyewall due to the low-level convergence therein (Kimball 2006). The air with higher $\theta_{e}$ is therefore not able to reach the inner core of TC and release the latent energy in the eyewall. Wang (2009) found a similar result that the heating from the outer rainband has negative impact on TC intensity. In addition, the larger surface fluxes in the outer region, implying larger lowlevel moisture there, are also found to reduce the TC intensity (Xu and Wang 2010; Ito et al. 2011).

For high $\theta_{e}$ to be favorable for TC intensification, we demonstrate that 5 times the RMW is the optimal critical distance from the center. This is also consistent with Wang (2009), who demonstrated that increasing (decreasing) the heating rate outside $90 \mathrm{~km}$ (about 6 times the RMW) from the storm center decreases (increases) the TC intensification rate. Therefore, increasing the $q_{v}$ (or $\theta_{e}$ ) in the boundary layer is only favorable for TC intensification rate when the increase of $\theta_{e}$ in the boundary layer is able to directly increase the $\theta_{e}$ in the eyewall.

\section{Concluding remarks}

This study aims to identify factors to which TC intensification rate is most sensitive, through convection-permitting ensemble simulations under idealized conditions without vertical wind shear and with fixed SST. As TC intensity is found to be a strong confounding variable in both TC structure and TC intensification rate, the partial correlation is used to control the effect of TC intensity when estimating the relationship between TC structure and its future intensification rate.

The partial correlations of the thermodynamic variables with the intensification rate are generally greater than the dynamical variables. This may be due to the fact that the important dynamical variables for the intensification rate are more related to the current intensity. This result is consistent with previous studies utilizing the adjoint method to identify the sensitivity since there is no inherent initial intensity uncertainty in the adjoint method. In particular, $\theta_{e}$ in the region from RMW to 3 times the RMW below $2 \mathrm{~km}$ has the largest correlation (over 0.7 ) with 2.5 -h intensity change. This implies that $\theta_{e}$ in that region can serve as a desirable precursor for the

TABLE 1. List of the verification experiments.

\begin{tabular}{|c|c|c|c|c|c|}
\hline Expt Name & Center $\left(r_{j}\right)$ & Magnitude & Expt name & Center $\left(r_{j}\right)$ & Magnitude \\
\hline CTRL & - & $0 \%$ & & & \\
\hline$P_{1}$ & RMW & $+10 \%$ & $N_{1}$ & RMW & $-10 \%$ \\
\hline$P_{2}$ & 2RMW & $+10 \%$ & $\mathrm{~N}_{2}$ & 2RMW & $-10 \%$ \\
\hline$P_{3}$ & 3RMW & $+10 \%$ & $\mathrm{~N}_{3}$ & 3RMW & $-10 \%$ \\
\hline$P_{4}$ & 4RMW & $+10 \%$ & $N_{4}$ & 4RMW & $-10 \%$ \\
\hline$P_{5}$ & 5RMW & $+10 \%$ & $\mathrm{~N}_{5}$ & 5RMW & $-10 \%$ \\
\hline$P_{6}$ & 6RMW & $+10 \%$ & $N_{6}$ & 6RMW & $-10 \%$ \\
\hline$P_{7}$ & 7RMW & $+10 \%$ & $N_{7}$ & 7RMW & $-10 \%$ \\
\hline$P_{8}$ & 8RMW & $+10 \%$ & $N_{8}$ & 8RMW & $-10 \%$ \\
\hline
\end{tabular}




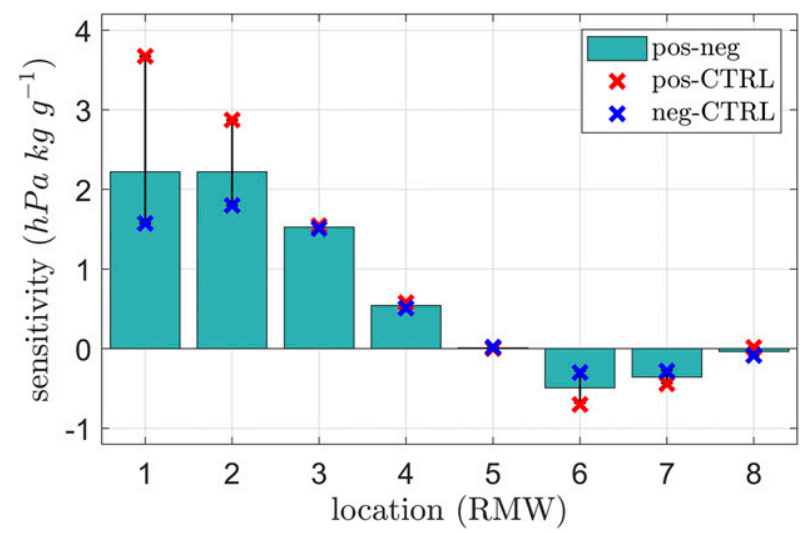

FIG. 17. The sensitivity $\left(\mathrm{hPa} \mathrm{kg} \mathrm{g}^{-1}\right)$ of the averaged minimum sea level pressure during $t=0-5 \mathrm{~h}$ to the water vapor perturbation at different locations. The bar is calculated from the difference between the positive experiment $\left(P_{i}\right)$ and the negative experiment $\left(N_{i}\right)$, the red cross is calculated from the difference between the positive experiment $\left(P_{i}\right)$ and the control experiment (CTRL), and the blue cross is calculated from the difference between the negative experiment $\left(N_{i}\right)$ and the control experiment (CTRL), where $i=1,2, \ldots, 8$.

change of TC intensification rate, if the initial intensity is well constrained.

With higher $\theta_{e}$ in the region from RMW to 3 times the RMW below $2 \mathrm{~km}, \theta_{e}$ above the boundary layer (around $3 \mathrm{~km}$ ) becomes larger due to the vertical advection. Higher $\theta_{e}$ there decreases the convective stability, causing the parcel to undergo an enhanced acceleration, which leads to a more upright updraft, and an inward shift of vertical motion and heating in mid- to upper eyewall. The upright trajectory of the parcels with enhanced vertical acceleration is more likely to increase the absolute angular momentum (AAM) in the eyewall by a small yet positive amount, as a result of the small imbalance between vertical and radial advection. The increase of AAM in the eyewall leads to the increase of inertial stability inside the eyewall. The larger heating and the inward location of heating together lead to the greater intensification rate. Although larger heating associated with higher $\theta_{e}$ in the boundary layer near eyewall is well known, one important finding highlighted here is that the location of heating in the mid- to upper eyewall is also influenced by the $\theta_{e}$ in the sensitive region.

It is found that the development of an outer rainband is most related to the decrease of $\theta_{e}$ in the region from RMW to 3 times the RMW below $2 \mathrm{~km}$. Trajectory analysis shows that the decreased $\theta_{e}$ is attributed to two reasons: 1) a larger portion of parcels reaching the sensitive region are from midlevel, and 2) the boundary layer inflow becomes cooler and this low $\theta_{e}$ is not able to be recovered by the surface flux before reaching the sensitive region, despite the larger air-sea contrast and the expansion of tangential wind associated with the rainband.

In our verification experiments, we find that the sensitivity of 2.5-h intensity change to $q_{v}$ at around RMW or 2 times the RMW is $2-3 \mathrm{hPa}$ for $5 \%-6 \% q_{v}$ change. We also find a linear response of TC intensification to $q_{v}$ at around 3-4 times the
RMW. In addition, it is found that 5 times the RMW is a critical distance within (outside) which the $q_{v}$ (or $\theta_{e}$ ) is positively (negatively) correlated with the future TC intensification rate. It is emphasized that increasing the $q_{v}$ (or $\theta_{e}$ ) in the boundary layer is only favorable for TC intensification rate when the increase of $\theta_{e}$ in the boundary layer is able to directly increase $\theta_{e}$ in the eyewall.

We note that our results are based on the idealized environmental conditions. TC intensification can be more complicated, such as when TC is embedded in a strong vertical wind shear environment (e.g., Zhang and Tao 2013; Zhou 2015). In this case, TC intensification may be more sensitive to some of the asymmetric structures. In addition, SST cooling is not considered in this study. However, we still consider that the sensitivity of TC intensification to $\theta_{e}$ will still be important regardless of the environmental conditions. Our hypothesis is that different environmental conditions can be reflected on the different $\theta_{e}$ distribution in the TC, while the positive impact of $\theta_{e}$ to the TC intensification may still be unchanged.

Acknowledgments. This work is supported by the Ministry of Science and Technology of Taiwan under Grants MOST 106-2111-M-002-013-MY3 and MOST 107-2111-M-002-016-MY3 and by the Office of Naval Research through Grants N62909-16-1-2169 and N00014-20-1-2467. Valuable comments from Kosuke Ito, Yi-Hsuan Huang, Dandan Tao, Michael Decaria, Peter Jan van Leeuwen, the editor, and three anonymous reviewers that helped improve the quality of the manuscript are highly appreciated.

\section{REFERENCES}

Alland, J. J., B. H. Tang, and K. L. Corbosiero, 2017: Effects of midlevel dry air on development of the axisymmetric tropical cyclone secondary circulation. J. Atmos. Sci., 74, 1455-1470, https://doi.org/10.1175/JAS-D-16-0271.1.

Brown, B. R., and G. J. Hakim, 2015: Sensitivity of intensifying Atlantic hurricanes to vortex structure. Quart. J. Roy. Meteor. Soc., 141, 2538-2551, https://doi.org/10.1002/qj.2540.

Carrasco, C. A., C. W. Landsea, and Y. Lin, 2014: The influence of tropical cyclone size on its intensification. Wea. Forecasting, 29, 582-590, https://doi.org/10.1175/WAF-D-13-00092.1.

Chang, C., and C. Wu, 2017: On the processes leading to the rapid intensification of Typhoon Megi (2010). J. Atmos. Sci., 74, 1169-1200, https://doi.org/10.1175/JAS-D-16-0075.1.

Corsaro, C., and R. Toumi, 2017: A self-weakening mechanism for tropical cyclones. Quart. J. Roy. Meteor. Soc., 143, 2585-2599, https://doi.org/10.1002/qj.3109.

DeMaria, M., M. Mainelli, L. K. Shay, J. A. Knaff, and J. Kaplan, 2005: Further improvements to the Statistical Hurricane Intensity Prediction Scheme (SHIPS). Wea. Forecasting, 20, 531-543, https://doi.org/10.1175/WAF862.1.

_ C. R. Sampson, J. A. Knaff, and K. D. Musgrave, 2014: Is tropical cyclone intensity guidance improving? Bull. Amer. Meteor. Soc., 95, 387-398, https://doi.org/10.1175/BAMS-D12-00240.1.

Didlake, A. C., and R. A. Houze, 2013: Dynamics of the stratiform sector of a tropical cyclone rainband. J. Atmos. Sci., 70, 18911911, https://doi.org/10.1175/JAS-D-12-0245.1.

Doyle, J. D., C. A. Reynolds, C. Amerault, and J. Moskaitis, 2012: Adjoint sensitivity and predictability of tropical cyclogenesis. 
J. Atmos. Sci., 69, 3535-3557, https://doi.org/10.1175/JAS-D12-0110.1.

Dudhia, J., 1989: Numerical study of convection observed during the Winter Monsoon Experiment using a mesoscale two-dimensional model. J. Atmos. Sci., 46, 3077-3107, https://doi.org/10.1175/15200469(1989)046<3077:NSOCOD > 2.0.CO;2.

Emanuel, K. A., 1991: The theory of hurricanes. Annu. Rev. Fluid Mech., 23, 179-196, https://doi.org/10.1146/annurev.fl.23. 010191.001143.

—_, and F. Zhang, 2016: On the predictability and error sources of tropical cyclone intensity forecasts. J. Atmos. Sci., 73, 37393747, https://doi.org/10.1175/JAS-D-16-0100.1.

— and — 2017: The role of inner-core moisture in tropical cyclone predictability and practical forecast skill. J. Atmos. Sci., 74, 2315-2324, https://doi.org/10.1175/JAS-D-17-0008.1.

Hong, S., Y. Noh, and J. Dudhia, 2006: A new vertical diffusion package with an explicit treatment of entrainment processes. Mon. Wea. Rev., 134, 2318-2341, https://doi.org/10.1175/ MWR3199.1.

Ito, K., Y. Ishikawa, Y. Miyamoto, and T. Awaji, 2011: Short-timescale processes in a mature hurricane as a response to sea surface fluctuations. J. Atmos. Sci., 68, 2250-2272, https:// doi.org/10.1175/JAS-D-10-05022.1.

Jordan, C. L., 1958: Mean soundings for the West Indies area. J. Meteor., 15, 91-97, https://doi.org/10.1175/1520-0469(1958) 015<0091:MSFTWI > 2.0.CO;2.

Kimball, S. K., 2006: A modeling study of hurricane landfall in a dry environment. Mon. Wea. Rev., 134, 1901-1918, https://doi.org/ 10.1175/MWR3155.1.

Liu, S., D. Tao, K. Zhao, M. Minamide, and F. Zhang, 2018: Dynamics and predictability of the rapid intensification of Super Typhoon Usagi (2013). J. Geophys. Res. Atmos., 123, 7462-7481, https://doi.org/10.1029/2018JD028561.

Minamide, M., and F. Zhang, 2018: Assimilation of all-sky infrared radiances from Himawari- 8 and impacts of moisture and hydrometer initialization on convection-permitting tropical cyclone prediction. Mon. Wea. Rev., 146, 3241-3258, https:// doi.org/10.1175/MWR-D-17-0367.1.

Miyamoto, Y., and T. Takemi, 2013: A transition mechanism for the spontaneous axisymmetric intensification of tropical cyclones. J. Atmos. Sci., 70, 112-129, https://doi.org/10.1175/ JAS-D-11-0285.1.

Mlawer, E. J., S. J. Taubman, P. D. Brown, M. J. Iacono, and S. A. Clough, 1997: Radiative transfer for inhomogeneous atmospheres: RRTM, a validated correlated-k model for the longwave. J. Geophys. Res., 102, 16 663-16682, https://doi.org/ 10.1029/97JD00237.

Molinari, J., J. Frank, and D. Vollaro, 2013: Convective bursts, downdraft cooling, and boundary layer recovery in a sheared tropical storm. Mon. Wea. Rev., 141, 1048-1060, https:// doi.org/10.1175/MWR-D-12-00135.1.

Nystrom, R. G., F. Zhang, E. B. Munsell, S. A. Braun, J. A. Sippel, Y. Weng, and K. Emanuel, 2018: Predictability and dynamics of Hurricane Joaquin (2015) explored through convectionpermitting ensemble sensitivity experiments. J. Atmos. Sci., 75, 401-424, https://doi.org/10.1175/JAS-D-17-0137.1.

Poterjoy, J., and F. Zhang, 2011: Dynamics and structure of forecast error covariance in the core of a developing hurricane. J. Atmos. Sci., 68, 1586-1606, https://doi.org/10.1175/2011JAS3681.1.

Rogers, R., P. Reasor, and S. Lorsolo, 2013: Airborne Doppler observations of the inner-core structural differences between intensifying and steady-state tropical cyclones. Mon. Wea. Rev., 141, 2970-2991, https://doi.org/10.1175/MWR-D-12-00357.1.
Rotunno, R., and K. A. Emanuel, 1987: An air-sea interaction theory for tropical cyclones. Part II: Evolutionary study using a nonhydrostatic axisymmetric numerical model. J. Atmos. Sci., 44, 542-561, https://doi.org/10.1175/1520-0469(1987)044<0542: AAITFT $>2.0 . \mathrm{CO} ; 2$.

Schubert, W. H., and J. J. Hack, 1982: Inertial stability and tropical cyclone development. J. Atmos. Sci., 39, 1687-1697, https:// doi.org/10.1175/1520-0469(1982)039<1687:ISATCD>2.0.CO;2.

Sippel, J. A., and F. Zhang, 2008: A probabilistic analysis of the dynamics and predictability of tropical cyclogenesis. J. Atmos. Sci., 65, 3440-3459, https://doi.org/10.1175/2008JAS2597.1.

- , and — 2010: Factors affecting the predictability of Hurricane Humberto (2007). J. Atmos. Sci., 67, 1759-1778, https://doi.org/10.1175/2010JAS3172.1.

Tang, B., and K. Emanuel, 2010: Midlevel ventilation's constraint on tropical cyclone intensity. J. Atmos. Sci., 67, 1817-1830, https://doi.org/10.1175/2010JAS3318.1.

$\longrightarrow$, and - 2012: Sensitivity of tropical cyclone intensity to ventilation in an axisymmetric model. J. Atmos. Sci., 69, 23942413, https://doi.org/10.1175/JAS-D-11-0232.1.

Tao, D., and F. Zhang, 2014: Effect of environmental shear, seasurface temperature, and ambient moisture on the formation and predictability of tropical cyclones: An ensemble-mean perspective. J. Adv. Model. Earth Syst., 6, 384-404, https:// doi.org/10.1002/2014MS000314.

—, M. Bell, R. Rotunno, and P. J. van Leeuwen, 2020: Why do the maximum intensities in modeled tropical cyclones vary under the same environmental conditions? Geophys. Res. Lett., 47, e2019GL085980, https://doi.org/10.1029/2019GL085980.

Tao, W., J. Simpson, and M. McCumber, 1989: An ice-water saturation adjustment. Mon. Wea. Rev., 117, 231-235, https://doi.org/ 10.1175/1520-0493(1989)117<0231:AIWSA>2.0.CO;2.

Torn, R. D., and D. Cook, 2013: The role of vortex and environment errors in genesis forecasts of Hurricanes Danielle and Karl (2010). Mon. Wea. Rev., 141, 232-251, https://doi.org/ 10.1175/MWR-D-12-00086.1.

Van Sang, N., R. K. Smith, and M. T. Montgomery, 2008: Tropical cyclone intensification and predictability in three dimensions. Quart. J. Roy. Meteor. Soc., 134, 563-582, https://doi.org/ 10.1002/qj.235.

Velden, C., and Coauthors, 2006: The Dvorak tropical cyclone intensity estimation technique: A satellite-based method that has endured for over 30 years. Bull. Amer. Meteor. Soc., 87, 1195-1210, https://doi.org/10.1175/BAMS-87-9-1195.

Vigh, J. L., and W. H. Schubert, 2009: Rapid development of the tropical cyclone warm core. J. Atmos. Sci., 66, 3335-3350, https://doi.org/10.1175/2009JAS3092.1.

Wang, H., and Y. Wang, 2014: A numerical study of Typhoon Megi (2010). Part I: Rapid intensification. Mon. Wea. Rev., 142, 2948, https://doi.org/10.1175/MWR-D-13-00070.1.

Wang, Y., 2009: How do outer spiral rainbands affect tropical cyclone structure and intensity? J. Atmos. Sci., 66, 1250-1273, https://doi.org/10.1175/2008JAS2737.1.

Wu, S., and B. J. Soden, 2017: Signatures of tropical cyclone intensification in satellite measurements of ice and liquid water content. Mon. Wea. Rev., 145, 4081-4091, https://doi.org/ 10.1175/MWR-D-17-0046.1.

Xu, J., and Y. Wang, 2010: Sensitivity of tropical cyclone inner-core size and intensity to the radial distribution of surface entropy flux. J. Atmos. Sci., 67, 1831-1852, https://doi.org/10.1175/ 2010JAS3387.1.

— tropical cyclone intensification rate on the storm intensity and 
size in the North Atlantic. Wea. Forecasting, 30, 692-701, https://doi.org/10.1175/WAF-D-14-00141.1.

- and — 2018: Dependence of tropical cyclone intensification rate on sea surface temperature, storm intensity, and size in the western North Pacific. Wea. Forecasting, 33, 523-537, https://doi.org/10.1175/WAF-D-17-0095.1.

Yamaguchi, M., J. Ishida, H. Sato, and M. Nakagawa, 2017: WGNE intercomparison of tropical cyclone forecasts by operational NWP models: A quarter century and beyond. Bull. Amer. Meteor. Soc., 98, 2337-2349, https://doi.org/10.1175/BAMS-D-16-0133.1.

Ying, Y., and Q. Zhang, 2012: A modeling study on tropical cyclone structural changes in response to ambient moisture variations.
J. Meteor. Soc. Japan, 90, 755-770, https://doi.org/10.2151/ JMSJ.2012-512.

Yu, C., and A. C. Didlake, 2019: Impact of stratiform rainband heating on the tropical cyclone wind field in idealized simulations. J. Atmos. Sci., 76, 2443-2462, https://doi.org/10.1175/JAS-D-18-0335.1.

Zhang, F., and D. Tao, 2013: Effects of vertical wind shear on the predictability of tropical cyclones. J. Atmos. Sci., 70, 975-983, https://doi.org/10.1175/JAS-D-12-0133.1.

Zhou, W., 2015: The impact of vertical shear on the sensitivity of tropical cyclogenesis to environmental rotation and thermodynamic state. J. Adv. Model. Earth Syst., 7, 1872-1884, https:// doi.org/10.1002/2015MS000543. 